\title{
Méthode graphique \\ pour le calcul des cheminées d'équilibre
}

\section{Graphical method for the calculation of surge tanks}

\author{
par M. BOUVARD ers J. MOLBERT

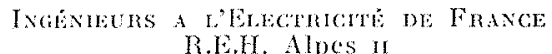

(Suite)

English synopsis, p. 372.

Dans un précédent numéro de la Houille Blanche, nous avons indiqué une méthode de calcul des cheminées d'équilibre, dont le principe a été défini par Schorrussch, généralisé cette méthode au cas où la cheminée est alimentée par des galeries en $\mathrm{V}$ et en $\mathrm{Y}$, et mis en évidence la notion de « hauteur accélératrice », notion générale qui, à notre avis, depasse le cadre de la méthode exposée. Nous nous proposons de poursuivre cetle étude (que nous supposerons connue du lecteur) :

\section{$1^{\circ}$ En appliquant cetle méthode :}

a) Au cas plus général où plusieurs cheminées sont insérées en série sur la galeric d'amenée. (Nous présenterons l'application pratique que nous en avons fait à un cas plus simple et plus courant où le nombre de ces cheminées est réduit à deux.)

b) Au cas où, du fait de la turbine, il existe une relation entre la montée deau dans la cheminée d'équilibre el le débit passant dans la turbine.

2" En indiquant ane manière nouvelle de prenare le probleme de la détermination d'une chemince d'équilibre qui permet d'en tronver sans tatonnement les dimensions optimum. La méthode graphique, en particulier, se prète tres bien a une telle eitude.

C'est ce que nous appellerons les procédés directs et rétrogrades.

\section{I. - Autres applications de la méthode graphique}

Nous allons appliquer la méthode graphique aux deux problemes suivants :
A) Cas de plusteurs cheminées en stirte: BRANCHÉES SUR UNE MÊME GALERTE D'AMENRE

Soit $n$ cheminées d'équilibre $C_{1}, \ldots C_{n}$ branchées en série sur une mêne galerie d'amence.

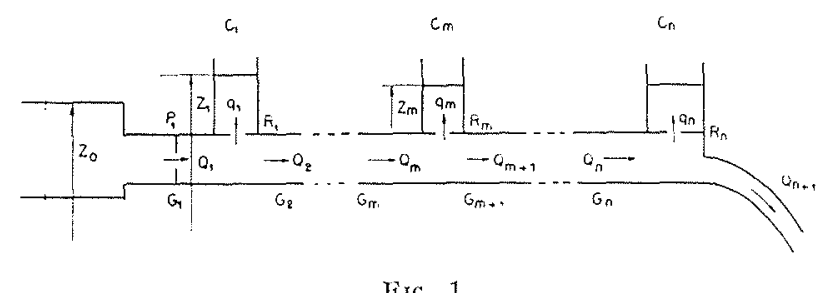

Désignons par :

$Q_{m}=$ le débit passant par la galerie $G_{m}$.

$q_{m}=$ le débit passant dans la cheminée $C_{m}$.

$Q_{(n, 1)}=$ débit passant dans la conduite forcée.

$Z_{0}=$ la cote de la prise d'eau.

$Z_{m}=$ la cote d'eau dans la cheminée $\mathrm{C}_{m}$.

$Z_{(n+1)}=$ la charge alu droil des turbines.

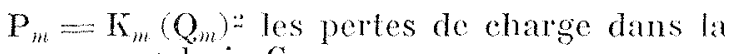
galerie $G_{m}$.

$\mathrm{P}_{(n+1)}=\mathrm{K}_{(n+1)}\left(\mathrm{Q}_{n+1}\right)^{2}$ les pertes de charge $\mathbf{R}_{m}=\mathrm{K}_{m}^{\prime}\left(q_{m}\right)^{2}$ les pertes de charge dans

Le mouvement de l'eau dans chacune des cheminées sera déterminé séparćment sur le graphique habituel par une courbe donnant son niveau en fonction du débit de la galerie située immédiatement à l'amont (fig. 2). Pour chaque système galerie-cheminée d'équilibre ainsi défini, le N.S. à la prise d'eau sera remplacé par le niveau piézométrique dans la galerie d'amenée au droit de licheming précédente. 
Donc, pour chaque système, nous tracerons les courbes caractéristiques (1), (2), (3), (4), et éventuellement (5), définies dans notre première citude.

Par exemple, la cheminé $\mathrm{C}_{m}$ recevra, en régime transitoire, le débit $q_{m}$ et son niveau d'eau sera représenté sur le graphique en fonction du (lébit $Q_{n}$ de la galcrie précédente $G_{m}$. On prendrat comme origine (variable) des niveaux, to niveau piézométrique dans la galerie au droit de la cheminée $\mathrm{C}_{(m-1)}$.

Les caractéristiques de la cheminée $\mathrm{C}_{m}$ seront déterminées par les courbes (3) $m-(4)_{m}-[$ Courbe

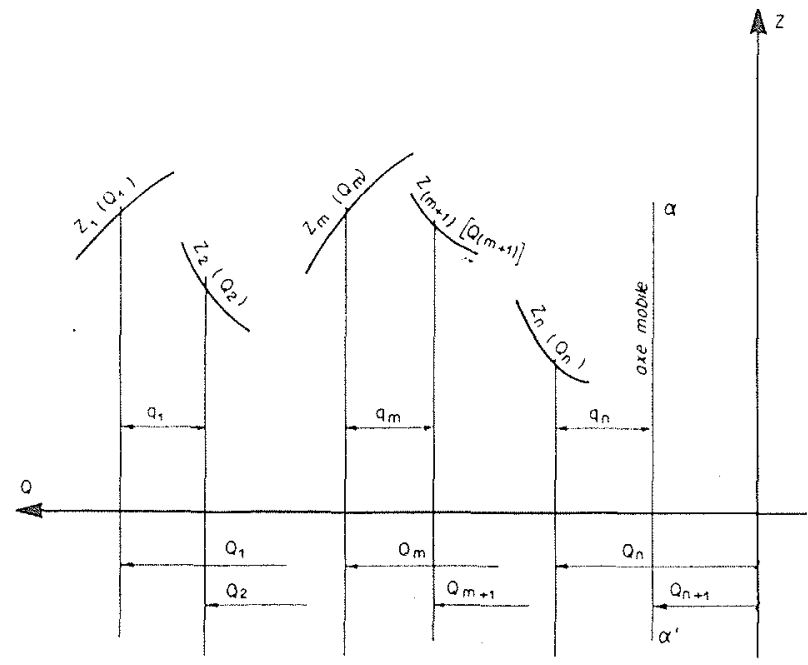

Fig. 2

des volumes] et éventuellement (1) bétrangloment à la base].

La galerie $G_{m}$ sera caractérisée par une parabole de pertes de charge $\mathrm{P}_{m}=\mathrm{K}_{m}\left(\mathrm{Q}_{m}\right)^{2}$ et par la droite $(2)_{m}$.

\section{1) Méthode de premiexe approxination :}

Supposons connus, à l'instant $t$ en régime transitoire, tous les débits du système $Q_{1}-Q_{2}$ etc. ..... $\mathrm{Q}_{(n+1)} ; q_{1}-q_{2}-$ cte. . . . $q_{n}$ et les niveaux à la prise d'eau el dans les $n$ cheminées d'équilibre $Z_{0}-Z_{1}-Z_{2 .} \ldots \ldots Z_{n}$ (fig. 2).

Nous pouvons déterminer les caractéristiques du système au temps $t+\Delta t, \Delta t$ étant suffisamment petit pour que les variations du systeme, durant cet intervalle de temps, puissent être considérées comme négligeables. Durant l'intervalle de temps $\Delta t$, les diverses grandeurs seront done supposées constantes et égales à leurs valeurs au temps $t$ que nous connaissons.

En effet, connaissant $q_{1}-q_{2}$, ete...... $q_{n}$, on peut calculer les montées d'eau élémentaires $\Delta Z_{1}, \Delta Z_{2}, \ldots \ldots \Delta Z_{n}$, grâce aux courbes $(3)_{1} \ldots \ldots$ $(3)_{n}$ et $(4)_{1} \ldots \ldots(4)_{n}$.

Connaissant, dans chaque galerie, Je débit et les pertes de charge au temps $t$, on peut calculer les hauteurs accélératrices correspondantes à chaque tronçon, $\mathrm{H}_{a_{1}} \ldots \ldots \mathrm{H}_{t n}(1)$. D'où les variations élémentaires de débit :

$$
\Delta Q_{1} \ldots \ldots \ldots \ldots Q_{n}
$$

données par les $n$ droites caractéristiques :

$$
(2)_{1} \ldots \ldots \ldots \ldots \ldots(2)_{n}
$$

Par exemple, reprenons la construction rela-

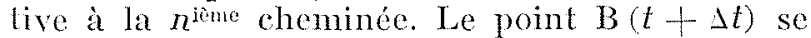
construit comme dans les epures habituelles, si l'on suppose que le niveau statique a la prise est remplacé par le niveau piézométrique au droit

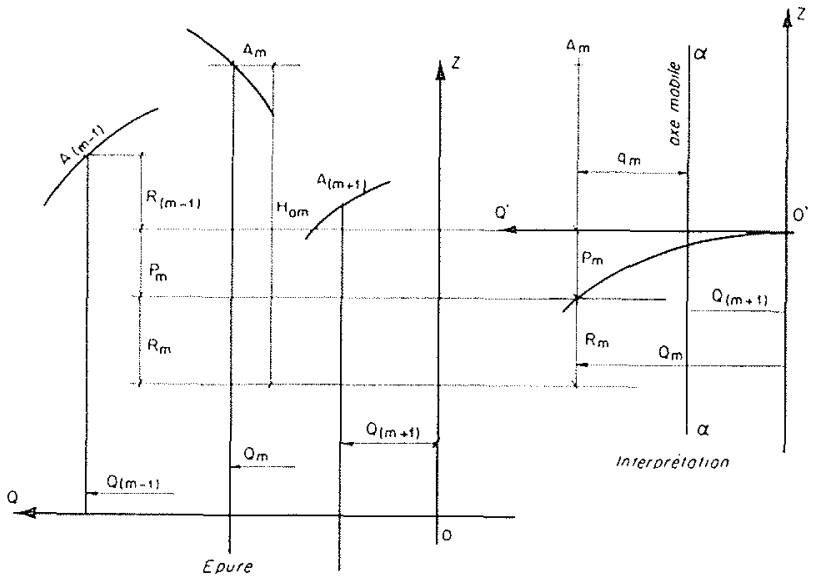

FIG. 4

de la cheminée précédente $\mathrm{C}_{(m-1)}$, et que l'axe des débits $Q_{(m+1)}$ représente l'axe mobile (fig. 4).

(1) Nous rappelons la définition de la hauteur accélératrice Soil par exemple la galerie $G_{m}$ limitée par les deux cheminées $\mathrm{C}_{(m-1,}$, et $\mathrm{C}_{m}$ (fig. 3). En régime transitoire, à l'instant $(i)$, les débits sont $q_{(m-1)}, Q_{m}$ et $q_{m}$.

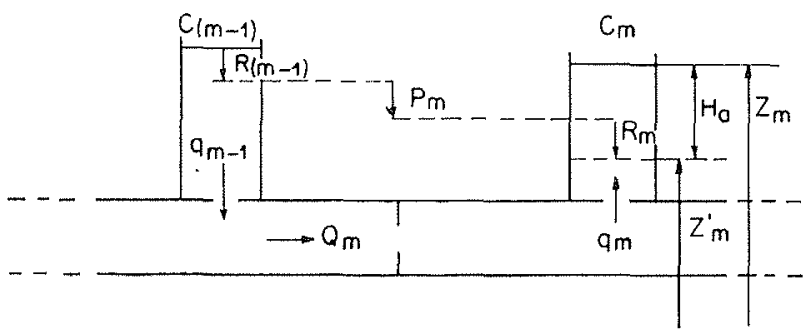

Fig. 3

Supposons qu'il existe un régime permanent caractérisé par ces trois débits, et tel que le niveau en $\mathrm{C}_{m-1}$, soit $Z_{(m-1)}$. Dans ce cas, le niveau dans la cheminée $C_{m}$ serait $Z_{m}^{\prime}$ qu'on deduirait facilement de $Z_{(m-1)}$ en retranchant, ou en ajoutant, les différentes pertes da charge.

La hanteur acceleratrice indiquant en quelque sorte l'élat de déséquilible du système a pour valeur :

$$
\mathrm{H}_{(m, m}=Z_{m}^{\prime}-Z_{m}
$$

L'intéret de cette notion provient du fait que toute la théorie des cheminées d'équilibre repose sur l'équation $\mathrm{Q}=\mathrm{o}_{a}$ : les variations de débits $\Delta \mathrm{Q}$ durant des intervalles de temps fixes $\Delta t$ sont proportionnelles à la hauteur accélératrice $\mathrm{H}_{a}$. 


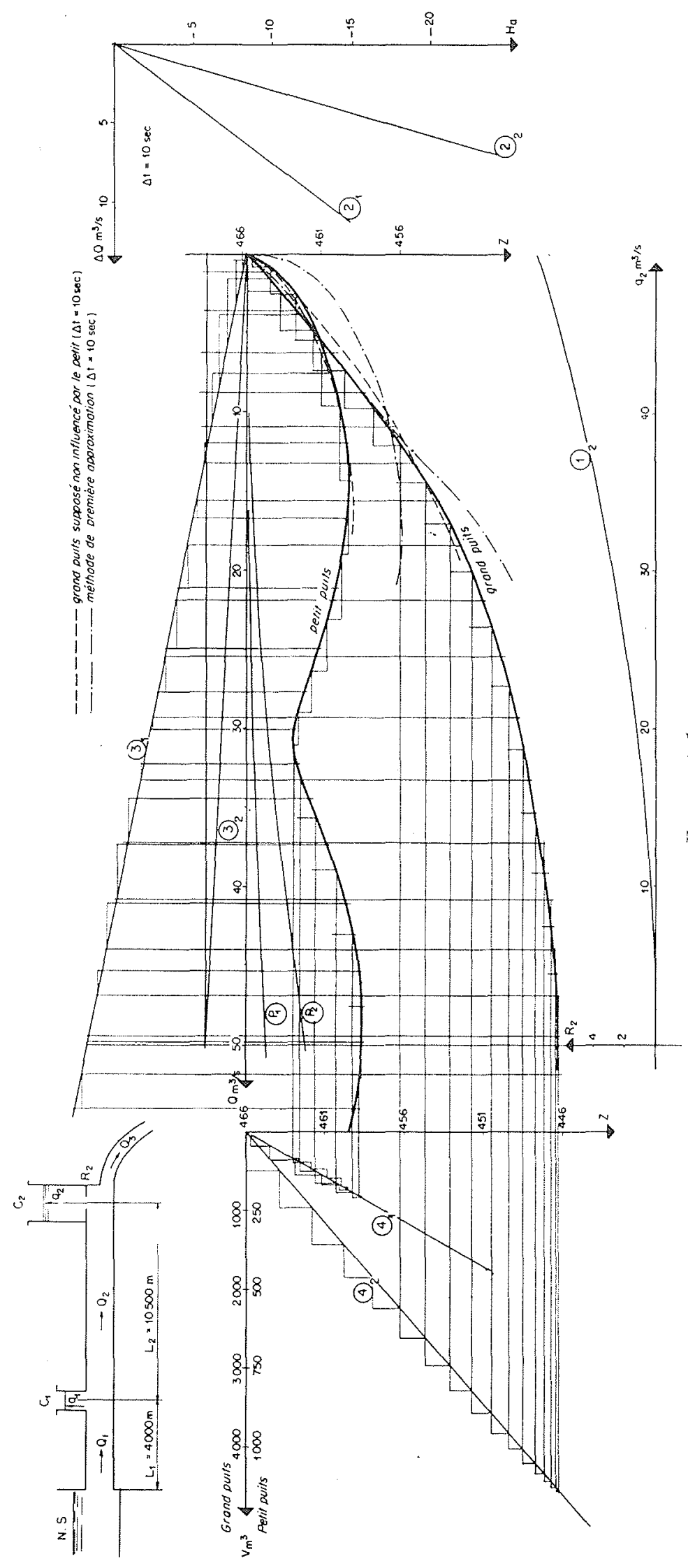

2) Méthode de deuxieme approximation :

Même construction en supputant d'abord le milieu de chaque segment élémentaire, puis en faisant la construction précédente à partir de ces milieux.

\section{3) Commencement d'une épure :}

Il est assez difficile, en général, de démarrer une épure car les segments correspondant aux premiers $\Delta t$ du mouvement transitoire sont très petits, et parce que la mise en mouvement dans les cheminées secondaires est relativement lente. Néanmoins, il est toujours possible de commencer correctement l'épure, au besoin en agrandissant la portion de graphique qui s'y rapporte, et en répétant plusieurs fois l'opération relative au premier $\Delta t$, le premier essai étant fait par la méthode de première approximation.

4) Cas particulier de deux cheminées d'équilibre, l'une étant de dimension nettement supérieure à l'autre :

Si l'une des deux cheminées est nettement plus petite que l'autre, on peut estimer que ces oscillations auront une influence négligeable sur la cheminée d'équilibre principale. Dans ce cas, on peut simplifier l'épure en procédant de la manière suivante :

- On fait d'abord l'épure relative à la cheminée d'équilibre principale supposée seule,

- On construit ensuite l'épure du petit puits a partir de celle de la cheminée d'équilibre principale.

Cette remarque simplifie beaucoup les épures quand on emploie la méthode de deuxième approximation. Il est évident qu'elle perd son intérêt si on emploic la méthode de premiere approximation qui est automatique, en ce sens qu'il n'y a pas à supputer les milieux de chaque segment. Les nombreuses épures que nous avons faites ont d'ailleurs prouvé que pour un $\Delta t$ donné, cette nouvelle approximation est beaucoup plus précise que la méthode de première approximation exécutée en tenant comple de la réaction du petit puits sur le grand.

\section{5) Application pratique (Epure $\mathrm{n}^{\circ} \mathrm{1}$ ) :}

Epure d'ouverture totale instantanée d'une chute possédant une cheminée d'équilibre et un puits de faible section situé au premier quart de la galerie d'amenée. 
Caractéristiques de l'installation

$$
\begin{aligned}
& \mathrm{Q}_{1}=\quad 50 \mathrm{~m}: \mathrm{s} \\
& \mathrm{L}_{1}=4.000 \mathrm{~m} \\
& \mathrm{I}=10.500 \mathrm{~m} \\
& f_{1}=f_{2}=31,1 \mathrm{~m}^{2} \\
& \mathrm{R}_{1}=0 \\
& R_{3}=7,5 \mathrm{~m} \text { pour } Q=50 \mathrm{~m} / \mathrm{s} \\
& \mathrm{P}_{1}=1,25 \mathrm{~m} \text { pour } \mathrm{Q}=50 \mathrm{~m} / \mathrm{s} \\
& \mathrm{P}_{2 .}=3,75 \mathrm{~m} \text { pour } \mathrm{Q}=50 \mathrm{~m} / \mathrm{s} \\
& \mathrm{Z}_{0}=4,66
\end{aligned}
$$

Le petit puits de $\varnothing 6 \mathrm{~m}$ est situé à $4 \mathrm{~km}$ de la prise d'eau. La chute étant relativement grande, l'influence des oseillations du plan d'eau sur lo débit turbiné est négligeable.

Nous avons fait l'épure par la méthode de deuxieme approximation ef nous avons marque en pointillé le résultat obtenu (tonjours avec le mème $\Delta t$ ) :

- avec la méthode de première approximation,

- en supposant auc la cheminée déquilibre principale n'est pas influencée par le petit puits.

La methode de premièe approximation domne des résultats absolument faux, il est probable qu'il faudrait, pour se rapprocher de la courbe exacte, prendre des $\Delta t$ cing a dix fois plus petits, ce qui rendrait l'épure extrêmement embrouillée.

L'autre approximation n'est pas mauvaise, comme le montre l'épure, mais il faut noter que l'erreur ne provient pas du choix de $\Delta t$ et qu'il est quelquefois assez difficile d'estimer à partir de quelle dimension le grand puits n'est pas influence d'une manière sensible par le petit puits.

B) CaS OU IL EXISTE UNE RELATION ENTRE

IE DÉBIT TURBINE ET LA HAUTEUR DE CHUTL:

Dans certains cas où les oscillations du plan d'eau dans la cheminée d'équilibre sont d'une amplitude non négligeable devant la hauteur de chute totale, le débit $Q_{(n+1)}$ qui passe dans les turbines à la fin d'une mancuvre de régulateur peut varier sensiblement au cours des oscillalions. Sans entrer dans le détail des nombreux cas qui peuvent se présenter suivant le type des turbines, le genre d'interconnexion, etc., la loi liant $Q_{(n+1)}$ à la charge $H$ au droit de la turbine [soit $\mathrm{Q}=f(\mathrm{H})$ ], peut toujours ètre représentéc sur le graphique habituel par la courbe $\Gamma$.

Le fait de tenir compte de la loi éventuelle. $\mathrm{Q}(\mathrm{H})$ ne complique pratiquement pas l'épure grâce à l'introduction de laxe mobile. En effet, la figure 5 montre la marche à suivre qui est très simple; à un instant donné $t$, laxe mobile passe par le point $M$ de la courbe $r$ qui a pour ordonnée la charge $Z_{(n+1)}$ au droit des turbines; cette charge $Z_{(\ldots 1)}$ se déduil immédiatement de $Z_{n}$ en retranchant, ou en ajoutant, suivant le cas, les pertes do charge dans l'etranglement et dans la conduite forcée.

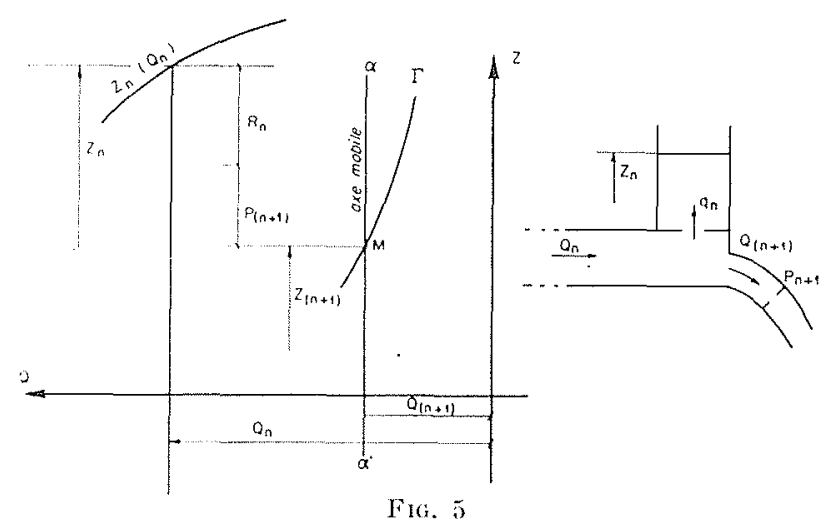

\section{ConchusION}

On pourrait certainement trouver d'autres exemples où l'application de la méthode graphique serait susceptible d'apporter rapidement une solution simple à des problèmes compliqués. Cela nous semble d'ailleurs parfaitement explicable, car le fonctionnement des cheminées d'équilibre peut, en fait, ètre séparé en deux phénomènes bien distincts :

$1^{\circ}$ Elles créent, dans la galerie sur laquelle elles sont insérées, mne accélération qui s'exprime tris simplement par :

$$
\Delta Q=\alpha \mathrm{H}_{i t} \Delta t
$$

2" Tant que le régime permanent n'est pas atteint, elles stockent (ou déstockent) la différence de débit entre l'amont et l'aval du point où elles sont insérées, ce qui s’exprime simplement par :

$$
\mathrm{F} d z=q d t
$$

Ces deux relations contiennent à elles seules la solution de tous les problèmes d'oscillation en masse. La facilité de la solution graphique provient du fait que ees relations restent distinctes, et aussi du fait que toutes les grandeurs intervenant dans les relations précédentes sont facilcment mesurables sur l'épure.

Les solutions analytiques obligent par contre à regrouper ces deux phénomènes, ce qui, même dans les cas simples, complique considérablement les calculs. 


\section{II. - Procédés rétrogrades et directes}

Dans ce chapitre, nous considérerons uniquement le système classique - prise d'eau unique - galerie --.- eheminée d'équilibre - représenté sur la figure 6.

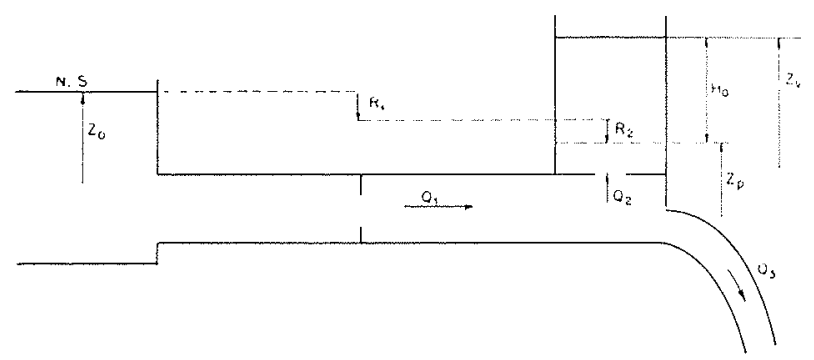

FIG. 6

Reprenons les notations que nous avions utilisées dans notre premier article. Nous les rappelons brièvement :

$\mathrm{F}=$ section de la cheminée d'équilibre.

$f=$ section de la galerie d'amenée.

$\mathrm{L}=$ longueur de la galerie d'amenée.

$Z_{0}=$ cote de l'eau à la prise d'eau (N.S.).

$Z_{\mathrm{v}}=$ cote de l'eau dans la cheminée en régime variable.

$Z_{\mathrm{p}}=$ cote de l'ean dans la cheminée en régime permanent fictif.

$Z_{\mathrm{m}}=$ cote maximum atteinte par l'eau aut cours d'une oscillation.

$Q_{1}, Q_{2}, Q_{3}=$ débits galerie, cheminée d'équilibre, conduite forcée.

$R_{1}=K_{1}\left(Q_{1}\right)^{2}=$ pertes de charge dans la galerie. $\mathrm{R}_{2}=\mathbf{K}_{2}\left(\mathrm{Q}_{2}\right)^{2}=$ perte de charge dans le diaphragme de la cheminée d'équilibre.

L'application du théorème des quantités de mouvement au fonctionnement du système oscillant en régime transitoire, jointe à l'équation de continuité appliquée à la galerie et à la cheminée d'équilibre, donne les relations suivantes :

$$
\left\{\begin{array}{l}
-\frac{g f}{\mathrm{~L}} \frac{d \mathrm{Q}_{1}}{d t}=Z_{0}+\mathrm{K}_{1}\left(\mathrm{Q}_{1}\right)^{2} \pm \mathrm{K}_{2}\left(\mathrm{Q}_{2}\right)^{2} \\
\mathrm{Q}_{2}=-\mathrm{F} d \mathrm{Z} \\
\mathrm{Q}_{1}+\mathrm{Q}_{2}=f(t, \mathrm{Z})
\end{array}\right.
$$

après élimination de $Q_{3}$.
Les caractéristiques de la chute $\mathrm{I}, f, \mathrm{R}_{1}$ sont données Nous supposerons $K_{2}$ constant (1)

La sconde équation permet de tenir compte des variations de débit non instantanées, à l'aval de la cheminée, et de la variation du débit turbiné en fonction de la cote de leau dans la cheminée d'équilibre

En régime transitoire, on peut considérer $Q_{1}$ Q, $-F$ et $Z$ comme des fonctions paramétriques de $t$. Elles ne peurent toutes etre déterminées par le systeme d'équation précident puisqu'on dispose de 3 equations pour 4 inconnues. Il faut done se fixer une quatrieme condition pour pouvoir résoudre le système. Habitucllement, on se fixe les caractéristiques de la cheminée, ce qui donne $F$ en fonction de $Z$. On détermine ensuite, en partant des conditions aux limites correspondant au régime permanent, la montée maximum de l'eau dans la cheminée d'équilibre, ce qui permet de vérifier si les caractéristiques de l'ouvage répondent aux conditions qu'il devra assurer.

\section{A) Procédé rétrograde}

\section{1) Exposé.}

Il est théoriquement possible de partir des conditions aux limites relatives à un point quelconque de fonctionnement en régime transitoire (notamment le point correspondant à la dénivellation maximum), pour en déduire le point de régime permanent d'où on est parti. C'est le procédé que nous appellerons « rétrograde», pour caractériser le fait que l'on remonte le temps.

Ce procédé peut être appliqué quel que soit le principe de calcul utilisé, qu'il soit analytique ou graphique. Nous allons uniquement exposer son utilisation dans le cadre de la méthode graphique.

\section{2) Utilisation du procédé rétrograde à laide de la méthode graphique.}

La méthode rétrograde consiste done à faire l'épure dans le sens des $t$ décroissants. Prenons, par exemple, l'etude d'une manouve d'onverture instantanée de $x$ à $100 \%$ (lig. 7 ).

Habituellement, on part du point A (point d'équilibre au début du mouvement) pour chercher, en particulier, le point $B$ minimum de l'oscillation.

(1) MM. Calame et Gaden ont précisé que, dans les chambres à étranglement, on aurait intérêt à faire varier $K_{i 2}$ pour obtenir une pression constante sous la cheminée d'écuilibre, mais ils ont admis que cela entraînerait des complications sans rappont avec le bénéfice retiré. 
L'épure se construit exactement de la mème manière, si on part de $\mathrm{B}$ (supposé connu) pour trouver le point A à l'intersection de la courbe $B A$ avec la parabole des pertes de charge lieu

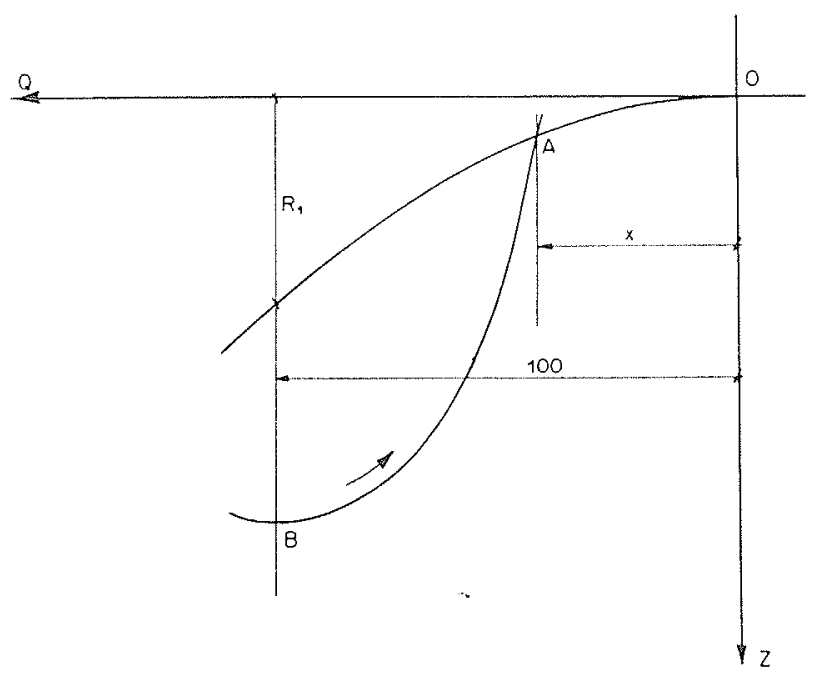

Fic. 7

des points de régime permanent de la cheminée en fonction du débit dans la galeric); il suffit simplement de porter les segments élémentaires $\Delta Z$ el $\Delta Q$ dans le sens opposé au sens habituel.

Remarque. - Cette méthode ne permet de considérer pratiquement que des manœurres d'ouverture ou de fermeture instantanés. En procédant par tâtonnements, on pourrait, sans doute, étudier des manouvres non instantanées; mais ces tatomnements feraient perdre a la méthode son principal intérèt qui est précisciment de les éviter.

\section{B) Procédé DrRecti}

\section{1) Exposé :}

Ainsi que nous l'avons vu, pour résoudre le systeme d'équation (1), il faut se donner une quatrieme relation entre les variables. Au lieu de se donner, comme on le lait habituellement, la section de la cheminée en fonction de la cote $Z$, il serait thériquement possible de se fixer n'importe quelle autre relation entre les variables. Beaucoup manqueraient de signification pratique et resteraient purement arbitraires ou sans objet. Cependant, comme nous allons le roir, certaines d'entre elles présenteraient un intérèt tout spécial. En effet, il serait particulièrement commode de résoudre le probleme en se fixant, à priori, la variation de la pression au bas de la cheminée d'équilibre $Z_{v}+K_{2}\left(Q_{2}\right)^{2}$ ou la variation du niveau dans la cheminée, et, en particulier, la valeur maximum de ces parametres.
On pourrait alors déterminer la cheminée d'équilibre par l'expression analytique ou graphique de la surface en fonction de l'altitude. Le problème des cheminées serait ainsi résolu d'une facon logique, puisquon déterminerait les caractéristiques de l'ouvrage à partir des conditions qu'il doit assurer.

C'est ce procédé que nous appellerons « procédé direct», pour caractériser le fait qu'on détermine «directement » la cheminée d'équilibre a partir des caractéristiques du fonctionnement qu'elle doit assurer.

Dans certains cas, le calcul permet de déterminer facilement les dimensions de la cheminéc, quand la relation de base est donnée sous une forme particulièrement simple.

Supposons que nous voulions caleuler une cheminée d'équilibre telle que, au cours d'une fermeture complète instantanée, la contre-pression dans la galerie au droit du puits reste constante durant la première montée de l'eau. Les équations se simplifient et deviennent :

$$
\begin{gathered}
\frac{\mathrm{L}}{g f} \frac{d \mathrm{Q}}{d t}=\mathrm{Z}+\mathrm{K}_{1}(\mathrm{Q})^{2}+\mathrm{K}_{2}(\mathrm{Q})^{2} \\
\mathrm{Q}=\mathrm{F} \frac{\mathrm{dZ}}{\mathrm{d} t}
\end{gathered}
$$

auxquelles il faudra ajouter la relation :

$$
Z+k_{2} Q_{2}=\text { Cste }=Z_{m}
$$

C'est, en effet, cette derniere valeur qui caractérise la pression au bas de la cheminće. Ce système simple s'intigre immediatement et donne, en posant :

$$
\frac{1}{\tau}=\frac{g f}{\mathrm{~L}} \sqrt{\mathrm{K}_{1} \mathrm{Z}_{m}}
$$

$$
\mathrm{Q}=\frac{-Z_{m} \operatorname{tg} \frac{t}{\tau}+\sqrt{\mathrm{K}_{1} Z_{m}} \mathrm{Q}_{0}}{\sqrt{\mathrm{K}_{1} Z_{m}}+\mathrm{K}_{1} \mathrm{Q}_{0} \operatorname{tg} \frac{t}{\tau}}
$$

et pour la surface de la chenince, en fonction de l'altitude $Z$ :

$$
\mathrm{F}=\frac{\mathrm{L}}{2 g f\left[\mathrm{~K}_{2} Z_{m}+\mathrm{K}_{1}\left(\mathrm{Z}_{m}-\mathrm{Z}\right)\right]}
$$

qui représente une hyperbole équilatère.

Le volume de la cheminée sobtient en intégrant l'expression diftérentielic FdZ entre - $R_{1}$ $\mathrm{ct}+Z_{m}$. On trouve, tous calculs faits :

$$
V=v \frac{W^{2}}{2 g} \frac{1}{\mathbf{R}_{1}} \log (1) \frac{\mathbf{K}_{2} Z_{m}+\mathbf{K}_{1}\left(Z_{m}+\mathbf{R}_{1}\right)}{\mathbf{K}_{2} Z_{m}}
$$

(1) log représentant lo symbole des logarithmes népericns pour eviter d'etre confondu avee L, longueur de Ia salerie d'amenée. 
(v) étant le volume de la galerie)

ou :

$$
\mathrm{V}=v \frac{\mathrm{W}^{2}}{2 g} \cdot \frac{1}{\mathrm{R}_{1}} \log \frac{Z_{m}+\mathrm{R}_{1}}{Z_{m}}
$$

Liexpression $b \frac{W^{2}}{2 g}$ represente linergie totale de la galerie au début du mouvement. Dans le cas où $R_{2}=R_{1}+Z_{13}$, on peul melle ecte formule sous la forme :

$$
\frac{V}{v}=\frac{W^{2}}{2 g} \frac{1}{R_{1}} \log (1)\left(1+\frac{R_{1}}{Z_{m}}\right)
$$

L'intérèt de celte formule vient de ce qu'elle donne facilement le volume minimum d'une chemince pour une contre-pression maximum donnće, et qu'elle permet de suive facilement leurs variations mutuelles.

Si $K_{1}$ est nul (pertes de charge dans la galerio nulles), on retrouve bien le cas theorique suivant lecuel la section constante assurerait une pression également constante. Elle en differe dautant plus que la perte de charge dans la galeric est plus importante.

Le calcul, qui permet de résondre le problème spécialement facile que nous venons de traiter, peut s'avérer incapable de traiter des cas un peu moins simples. Si la relation de base est plus compliquée, les équations différentielles risquent d'etre inintegrables, on tout au moins, difficiles à résoudre et à manier.

Nous allons voir que la méthode graphique permet de se donner facilement la relation do base, sous forme d'une courbe [dans le graphique habituel $\mathrm{Q}(\mathrm{H})$ ], quand celle-ci concerne la variation de pression au bas de la cheminćc ou du niveau d'eau, en fonction du débil dans la galerie. On peut alors construire facilement la cheminée donnée par l'expression $Z(V)$ sans avoir à se soucier de l'équation de la courbe de base qu'on s'est fixée.

Nous allons done exposer plus clairement cette dernière méthode en la comparant, dans les cas particuliers où c'est possible, à la méthode analytique.

\section{2) Utilisation du procédé direct à l'aide de la méthode graphique.}

Comme nous renons de l'exposer, le procedé direct consiste, chant donne :

- - les caracteristiques de la galerie [ courbes (1), (2), (3)],

-... la courbe $Z=f(Q)$,

à construire graphiquement la courbe (4) $Z=f(V)$, caractéristique de la cheminée, don- nant te volume $V$ en fonction de laltitude $Z$, de laquelle on déduit facilement la section $F$ en fonclion de $Z$.

\section{(1) Mithode de premiere approximation.}

Supposons la construction faile jusquan point $y_{1}$ de la courbe $Z=f(V)$ aucuel, an meme

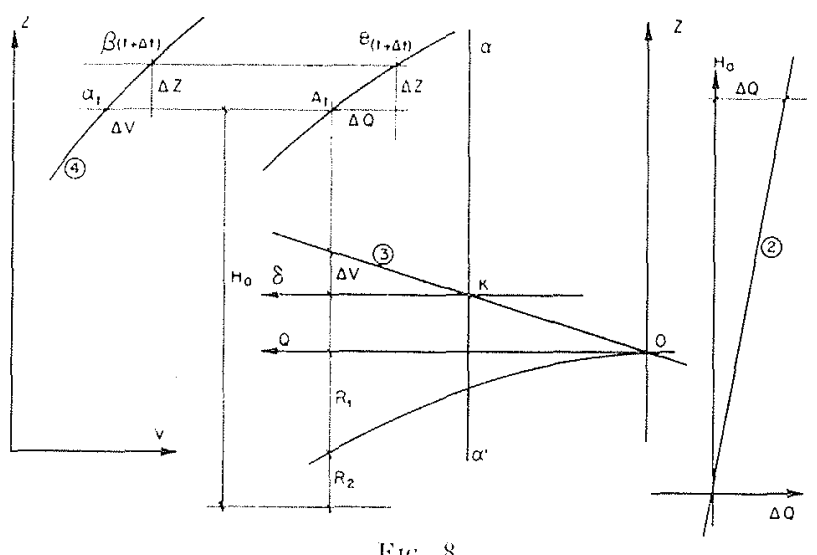

instant, correspond sur la meme ordonnce te point $A$, de la courbe $Z=f(Q)$ (lig. 8 ).

Pour un $\Delta t$ dome, nous allons ehereher successivement :

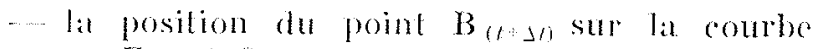
$Z=/(0)$

-... Te point $s(t) \Delta t)$ (qui sera un point de la courbe

$$
\left.Z=\int(r)\right) \text {. }
$$

$\Delta t$ dant un intervalle de temps suffisamment petit pour qu'on puisse considérer que $\mathrm{Q}_{2}$ et $\mathrm{H}_{t}$ soient constants durant cet intervalle et égaux a leurs valeurs au point $A_{1}$.

La connaissance du point A nous permet de trouver, sur le graphique, la quantité $\Delta T$ et la hauteur accélératrice $H_{4}$ (fig. 8), d'où la quantite

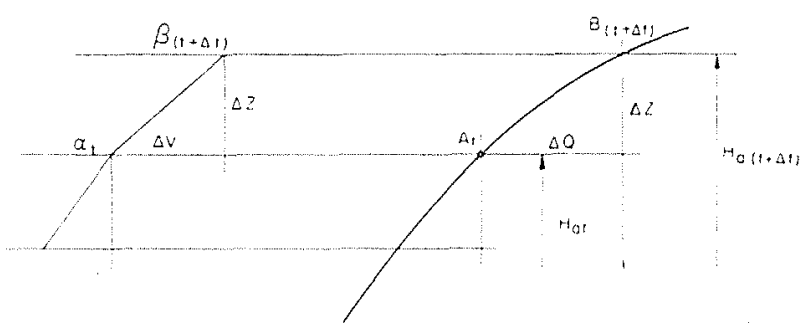

Fiti. !)

$\Delta Q . \Delta Q$ étant connue, le point $B_{(t+\Delta t j}$ est déterminé sur la courbe $Z=f(Q)$. co qui détermine, en même lemps, l'ordonnée du point $\beta_{(t+\Delta t)}$ sur la courbe $Z=f(V)$; IV ctant connu, le point $\beta_{(t ; \Delta t)}$ est déterminé.

On construit ainsi, point par point, de $\Delta t$ en $\Delta t$, la courbe $Z=f(V)$ (fig. 9 ). 
b) Wéthode de deuxieme approximation.

Même construction à partir du milicu estimć du fulur segment $A B$ (fig. 10).

La méthode de deuxième approximation est plus simple à employer dans ee cas que dans les

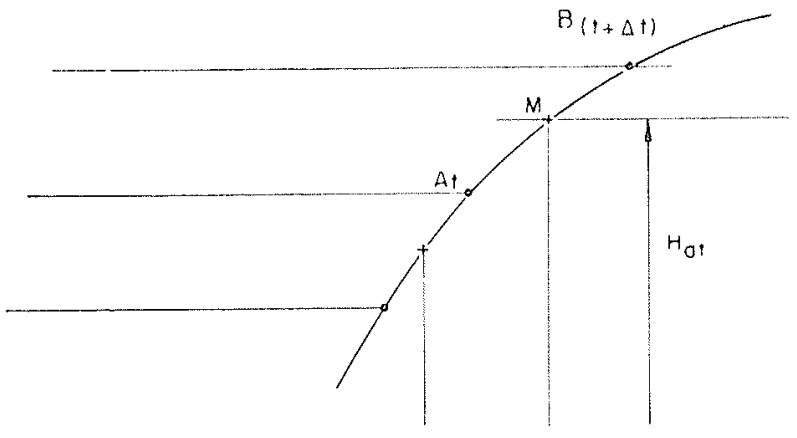

Fi(i. 10

épures classiques. Fn efret, dans celles-ci, on doit supputer a la fois la longueur et la direction du segment élémentaire suivant, alors que, dans la méthode directe, seule sa longueur est à estimer.

\section{c) Remarques}

Premiere rematrove. -... La collo des rolumes $Z=f(V)$ correspond à une forme de cheminée dans laquelle la section varierait d'une facon continue avec l'altitude; une telle cheminée serait, en sénéral, difficile, sinon impossible à construire pratiquement, les complications d'exécution pourant diminuer ou même rendre illusoire le bénéfice apporté par la réalisation idéale de la condition fixée. On peut cependant approcher de tres pres la forme donnée par l'épure, en remplaçant

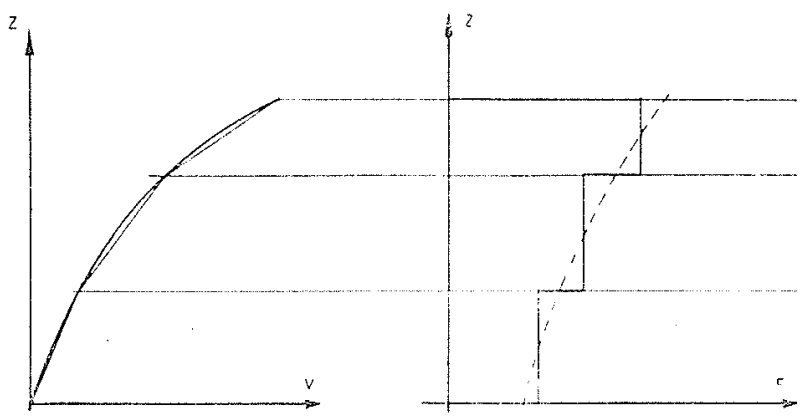

Fiti. 11

cette courbe $Z=f(V)$ par une ligne brisce; chaque segment correspondant à une portion de cheminéc à section constante (fig. 11).
DEUxibMe REMARQue.-Dans le cas d'une cheminée à étranglement, au lieu de se domner la courbe $Z=f(Q)$ relative au niveau de l'eau dans la chemince, on peut, et il est même préférable, de se donner la courbe $Z=f(Q)$ relative à la surpression sous l'étranglement. La courbe des niveaux s'en déduit d'ailleurs immédiatement en retranchant ou en ajoutent les pertes de charge de l'etranglement (supposées connues évidemment); on est ainst ramenc au probleme précedent.

Tromsine remaroue: Axe mobile.-- Rien n'empèche evidemment de considerer des manouveres de vannage non instantances, la construction n'étant pas, pour autant, plus compliquée que dans la móthode indirecte.

Qutrume memaroue : Procéde direct rétrograde. - Le procédé rétrograde peut évidemment être appliqué en même temps que le procédé direct; les épures se font de la même manière, que ce soit dans le sens des $t$ croissants que dans le sens des $t$ décroissants.

\section{C) RÉsumé DEs DIFrÉRENTS procédés JET DE LFURS COMBINAISONS}

Avant d'exposer quelques applications pratiques, nous allons passer en revuc les diffórents
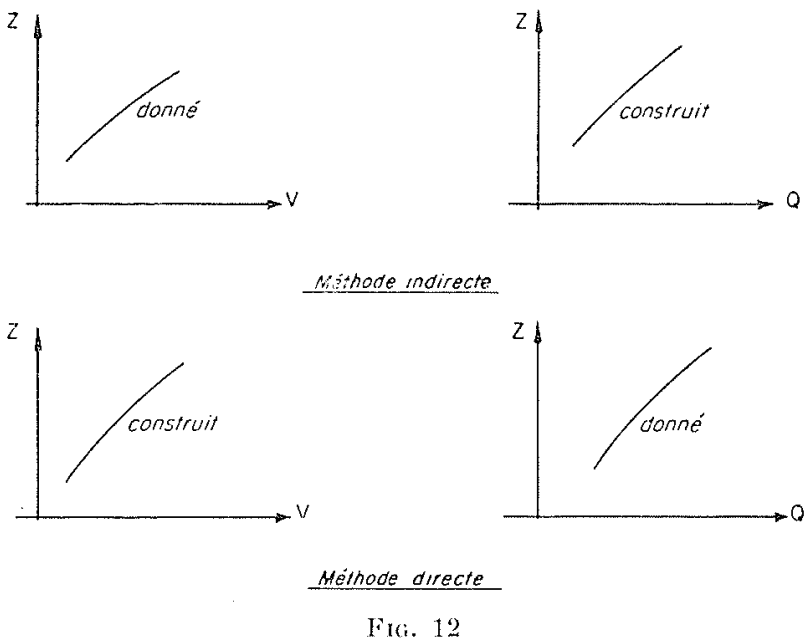

procédés d'étude dont nous venons de parler, ainsi que leurs combinaisons.

En se donnant $Z=f(\mathrm{~V})$, on peut construire $z=f(Q)$; c'est le procédé habituel indirect (ou indirect rétrograde si la courbe est construite dans le sens des $t$ dicroissants).

En se donnant $Z=f(Q)$, on peut construire $Z==f(V)$, c'est le procédé direct (ou direct rétrograde si la courbe est construite dans le sens des $t$ décroissants) (fig. 12). 
D'ailleurs, entre ces quatre méthodes, de nombreuses combinaisons sont possibles au cours d'une même épure. En effet, rien n'empèche de passer à un moment donné de la méthode directe à la méthode indirecte ou inversement (lig. 13).

Rien n'empêche également do commencer uno épure par deux extrémités à la fois, pour trouver

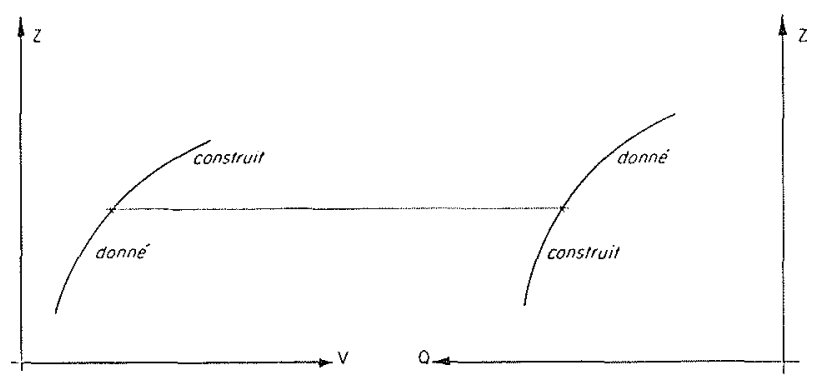

FIC. 13

le point d'intersection des deux courbes ainsi construites (fig. 14).

Toutes ces combinaisons peuvent sembler tres théoriques; elles permettent cependant de résou-

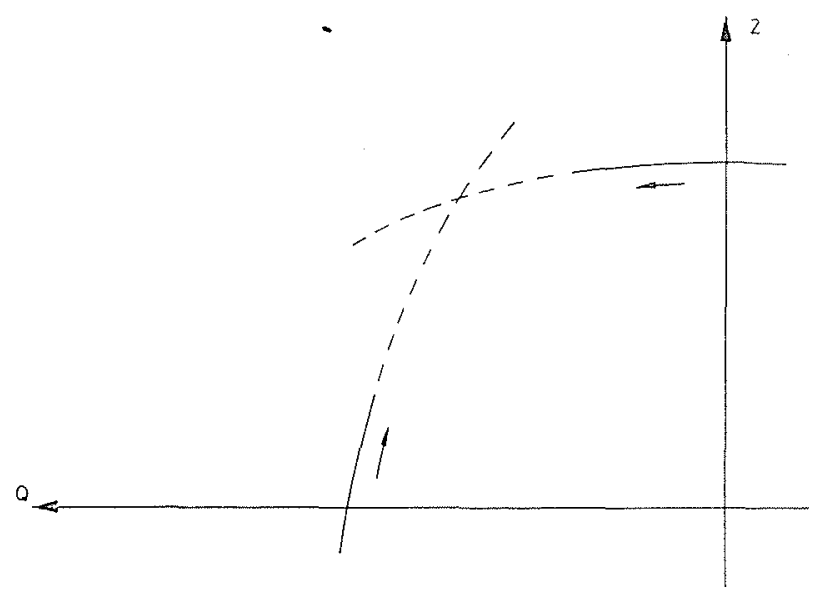

Fic. 14

dre élégamment un certain nombre de problèmes, dont nous allons donner quelques exemples.

\section{III. - Application pratique des procédés rétrogrades et directs}

Il serait fastidieux d'essayer de passer en revue tous les problemes particuliers qui se posent pour ehaque aménagement. Nous nous contenterons, à titre d'exemple, d'exposer quelques problèmes que nous avons en l'occasion d'étudier, en nous étendant cependant plus particulièrement sur le calcul des cheminées à étranglement et chambres d'expansion qui, à notre avis, présente un certain intérèt.

\section{A) Extmples diprication de prockint? RITROGRADE:}

a) Premier problime. - Soit un aménagement classique : prise d'eatunique - galerie - cheminée d'équilibre.

Les eaux sont captécs dans un réservoir à niveau variable.

Quelles sont les ouvertures de $x$ à $100 \%$ permises pour differents niveaux statiques it la retenue?

Le probleme se resoud sans tâtonnement par le procedé rétrograde (fig. 15). A chaque niveau statique correspond une difference de eote $\mathrm{H}$ en-

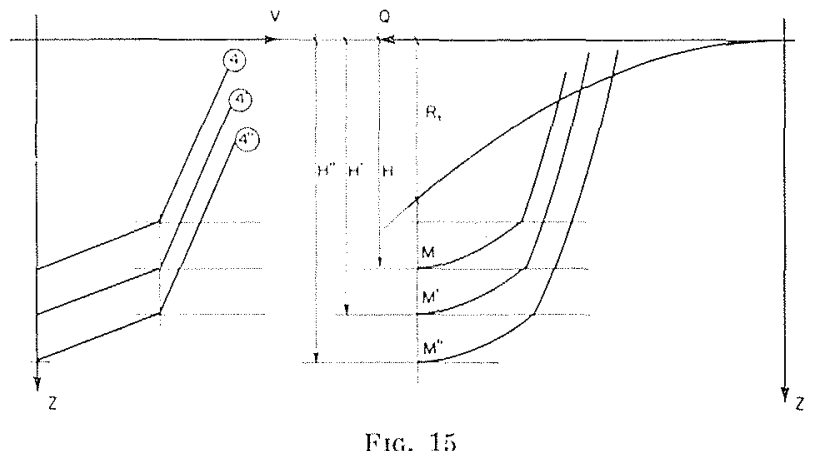

Fir. 15)

tre le fond de la chemince et le niveau statique. Il suffira de faire des épures dans se sens rétrograde, en prenant pour poinl de départ $\mathrm{M}, \mathrm{M}^{\prime}, \mathrm{M}^{\prime \prime}$ représentant pour chaque N.S. le point de cote minimum des courbes que l'on va construire. Labscisse des points d'intersection de ces courbes avec la parabole des pertes de charge (lien des points de régime permanent) domera les ouvertures permises.

\section{Application pratique (épure n" 2 ).}

Quelles sont les ouvertures permises pour les 3 N.S. : 471 - 468,5 - 466 d'une chute dont les caracteristiques sont les suivantes:

$$
\begin{aligned}
\mathrm{Q}_{1} & =100 \mathrm{~m} / \mathrm{s} \\
\mathrm{L} & =16.144 \mathrm{~m} \\
\mathrm{l} & =31,10 \mathrm{ml}^{2} \\
\mathrm{R}_{1} & =14 \mathrm{mpour} \mathrm{Q}=100 \mathrm{~m} \% / \mathrm{s} \\
\mathrm{R}_{2} & =30 \mathrm{~m} \text { pour } \mathrm{Q}=100 \mathrm{~m} / \mathrm{s}
\end{aligned}
$$

La cheminée d'ćquilibre est un puits eylindrique vertical de $\varnothing 17$ dont le fond est à la cote 440 .

Pour simplifier, sur l'épure, la construction 
n'est complète que pour la courbe relative au N.S. 471.

b) Deuxieme probleme. - On veut, pour des raisons constructives, que la cheminée d'équilibre soit formée de deux puits cylindriques superposés de diametres $\varnothing_{1}$ et $\varnothing_{2}$. A quelle cote doiton changer de diamètre, pour qu'au cours d'une fermeture totale (instantanée ou non) l'eau atteigne une cote maximum $\boldsymbol{Z}_{n}$ donnée?

Il suffira de commencer par deux extrémités,
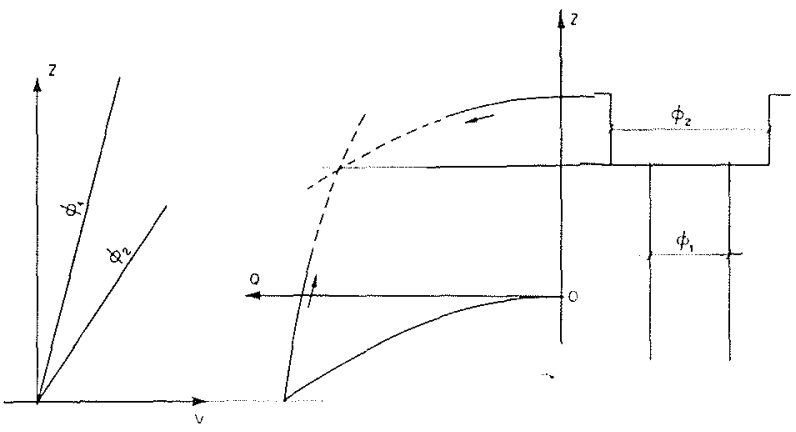

FIG. 16

comme indiqué sur la figure 16 ; l'intersection des deux courbes donnera la cote cherchée.

\section{c) Troisieme problème.}

Combien de temps le niveau dans la cheminée d'équilibre reste-t-il inférieur à une cote donnée au cours de l'oscillation la plus défavorable?

On commencera l'épure à partir de $M$, niveau minimum dans la cheminéc, dans deux direc-

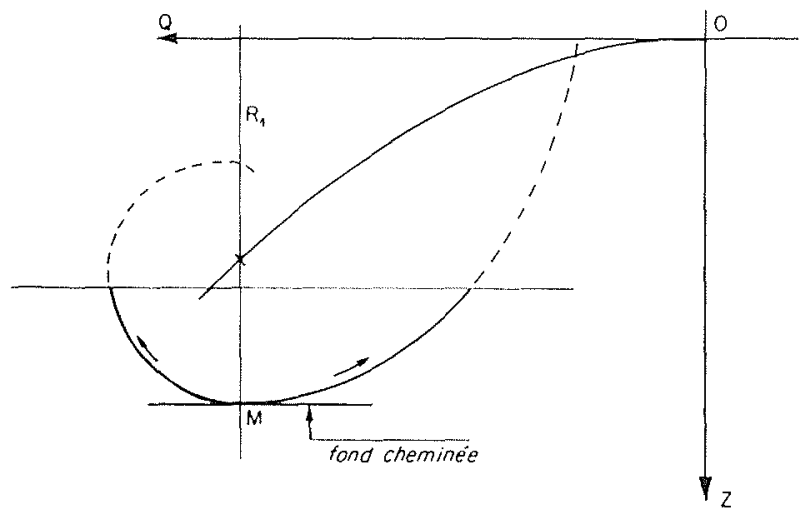

Fig. 17

tions a la fois, jusqu'à la cote fixce; il suffira de compter ensuite les $\Delta t$ (fig. 17).

B) Exmples dans lesoutes intervient LE PROCFIE DIRECT

a) Premier problème.--Détermination d'une chambre à étranglement optimum par le proeedé direct (fermeture totale instantanée).

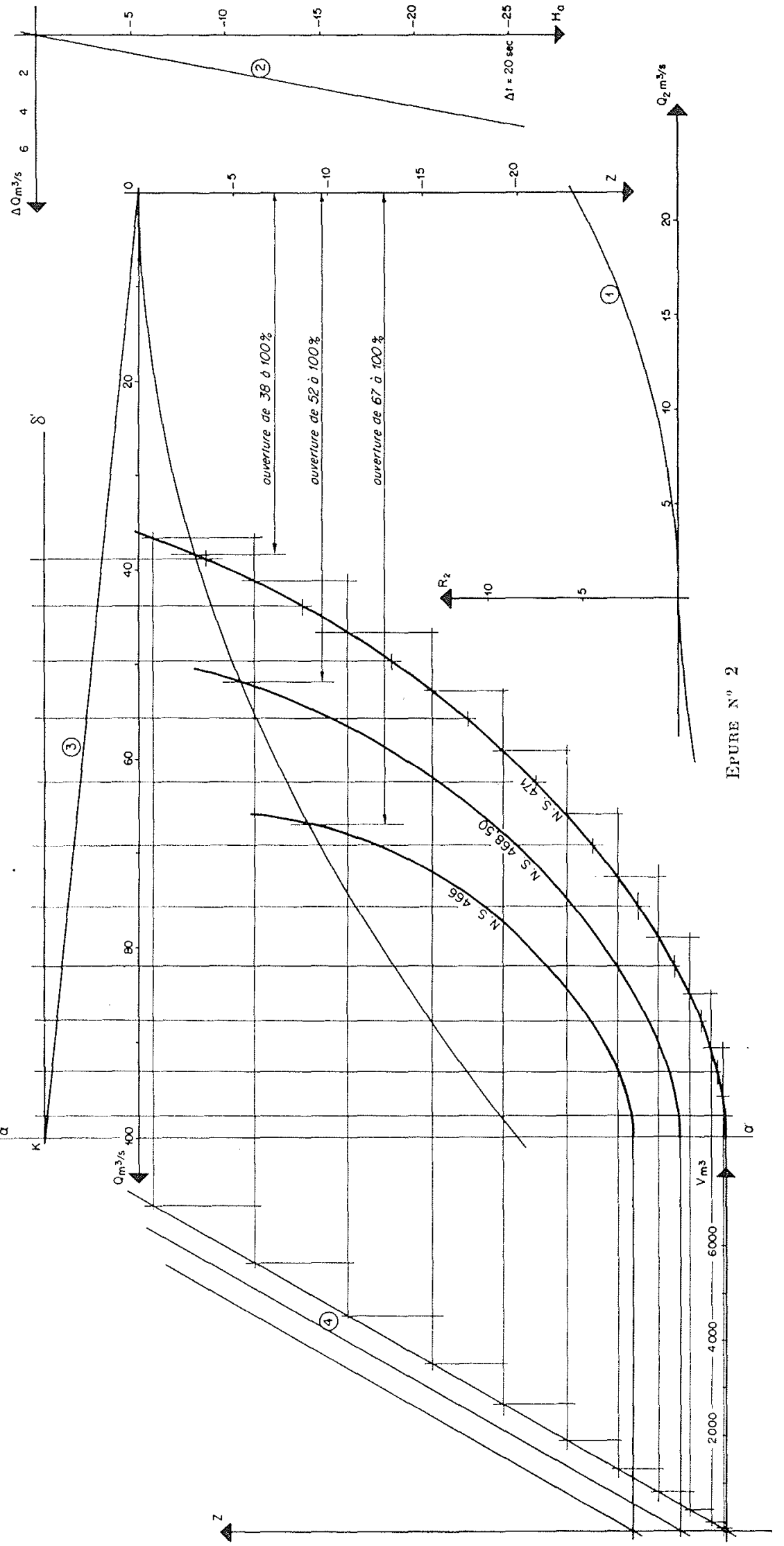




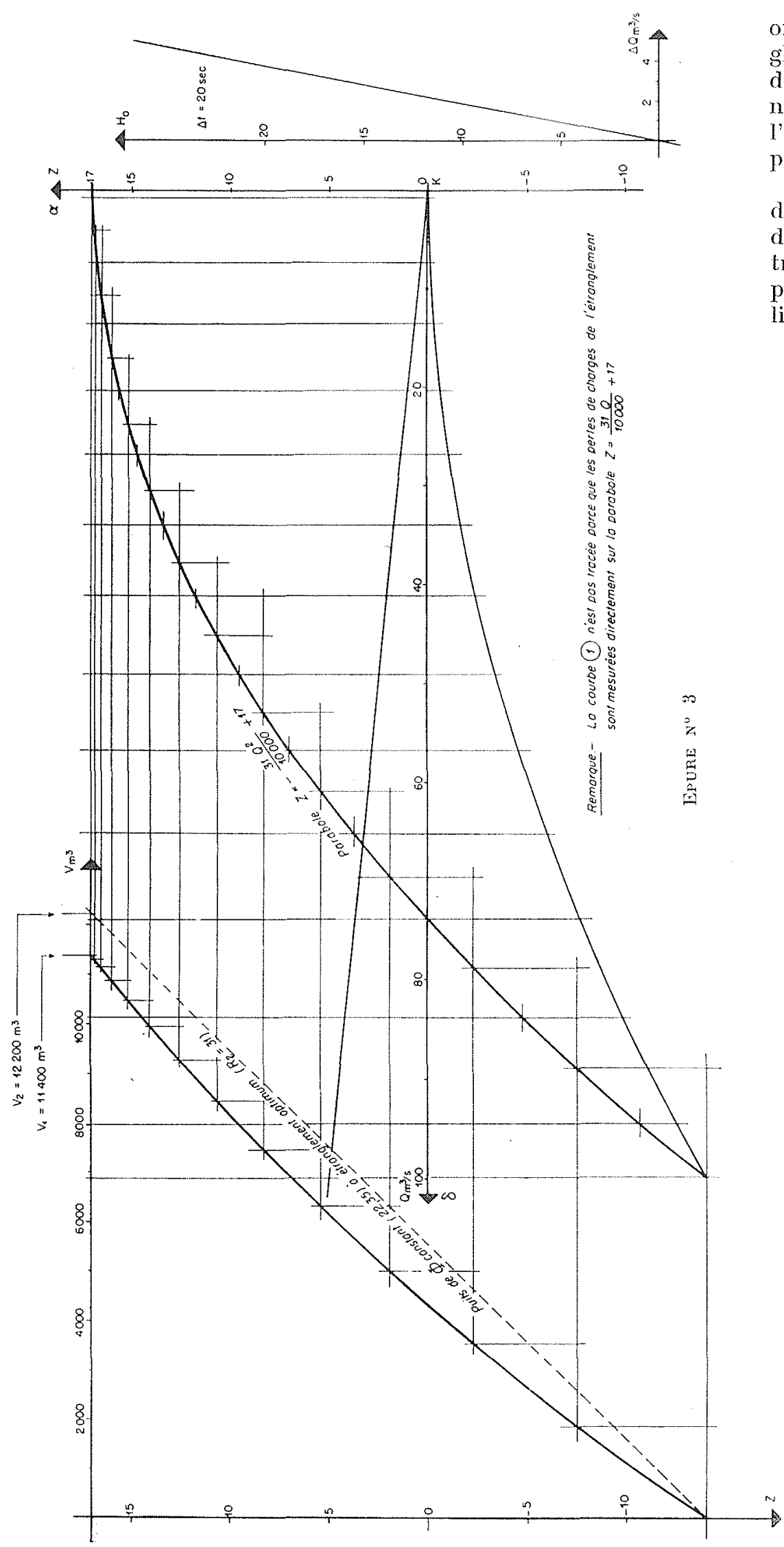

Outre les caractérisitques de la galerie, $\mathrm{L}, f, \mathrm{R}_{1}$, on se donne la surpression maximum sous étranglement, qui est supposée constante aul cours de la première montée de l'eau dans la cheminée d'équilibre. Si $Z_{m}$ est cette surpression, l'étranglement est défini et égal à $R_{2}=Z_{m}+R_{1}$ pour le débit maximum.

Comnaissant $R_{2}$, on peut tracer la parabole donnant le niveau de l'eau en fonction du débit dans la galerie. Par la méthode directe, on construit facilement la courbe $Z=f(\mathrm{~V})$, d'où on peut tirer la forme exacte de la cheminée d'équilibre (fig. 18).

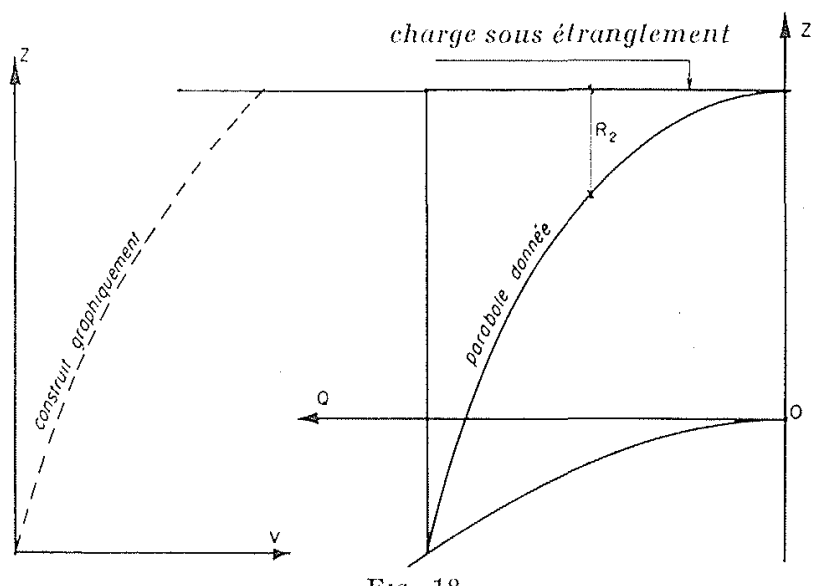

FIt. 18

Remarque. -.- Ya chemince eylindrique à diamètre constant, que donne l'application des abaques de Calame \& Gadren, suffit, en général, amplement pour calculer la cheminée, la contrepression étant presque constante. Cependant, l'application exacte de l'épure précédente peut être intéressante dans le cas des grosses cheminées pour lesquelles le puits unique à diamètre constant serait trop grand et devrait être remplacé par un ensemble de galeries. Dans ce cas, en jouant sur la longueur de ees galeries, on peut, à peu de frais, obtenir la contre-pression rigoureusement constante, et, par suite, une diminution de volume assez sensible.

\section{Application numérique (épure $n^{\circ} 3$ ).}

Epure de fermeture totale instantance d'une installation dont les caractéristiques sont les suivantes :

$$
\begin{aligned}
\mathrm{Q} & =100 \mathrm{~m}^{3} / \mathrm{s} \\
\mathrm{L} & =16.144 \mathrm{~m} \\
\mathrm{t} & =31,10 \mathrm{~m}^{2} \\
\mathrm{R}_{1} & =14 \mathrm{~m} \text { pour } \mathrm{Q}=100 \mathrm{~m}^{3} / \mathrm{s} \\
\mathrm{R}_{2} & =31 \mathrm{~m} \text { pour } \mathrm{Q}=100 \mathrm{~m}^{3} / \mathrm{s}
\end{aligned}
$$


Sur la courbe des volumes ainsi construite, on mesure, sur l'horizontale de cote $Z=17$, le volume de la cheminée d'équilibre compté à partir de la cote 14 , on trouve :

$$
\mathrm{V}_{1}=11.400 \mathrm{~m}^{3}
$$

La formule théorique précédente :

$$
\mathrm{V}=v \frac{\mathrm{W}^{2}}{2 \mathrm{~g}} \frac{1}{\mathrm{R}_{1}} \log \frac{\mathrm{Z}_{m}+\mathrm{R}_{1}}{\mathrm{Z}_{m}}
$$

donnerait également :

$$
\mathrm{V}_{1}=11,400 \mathrm{~m}^{3}
$$

ce qui est une bonne vérification de la précision de la méthode graphique.

La cheminée cylindrique à étranglement optimum, ayant la même montée max. $Z_{m}=17 \mathrm{~m}$, est facile à calculer à l'aide des abaques de MM. Callame \& Gaden; on trouve $\varnothing=22,35 \mathrm{~m}$, ce qui correspond à un volume :

$$
\mathrm{V}_{2}=12.200 \mathrm{~m}^{3}
$$

La différence relative :

$$
\frac{V_{2}-V_{1}}{V_{1}}=7,5 \%
$$

n'est pas négligeable et peut ètre intéressante à récupérer dans certains cas, comme nous l'avons indiqué dans la remarque précédente.

b) Denxième problème. - Calcul d'une cheminée à chambre d'expansion par le procédé direct et le procédé indirect combinés.

La galerie étant connue, on se donne isalement, en général :

- le diamètre du puits central, $\varnothing$,

- la hauteur des chambres d'expansion $h$ galerie d'un gabarit donné),

- les cotes maximum et minimum des oscillations de l'eau dans la cheminée d'équilibre, $Z_{m}$ et $Z_{m}^{\prime}$.

\section{Calcul d la fermeture}

On commence l'épure par la méthode directe (le puits central étant donné) jusqu'à la cote $Z_{i j}-h$; soit $M$ le point ainsi défini. On se donne une courbe MN a tangente horizontale en N re- présentant la fin de la montée de l'eau. On en déduit la courbe $Z=f(V)$ donnant la forme de l'expansion (fig. 19).

Remarque. - Une infinité de courbes satisfaisant à ces conditions peuvent être choisies; la plupart conduiraient évidemment à des courbes $Z(V)$ bizarres. L'expérience montre que la courbe de montée d'eau classique dans un puits

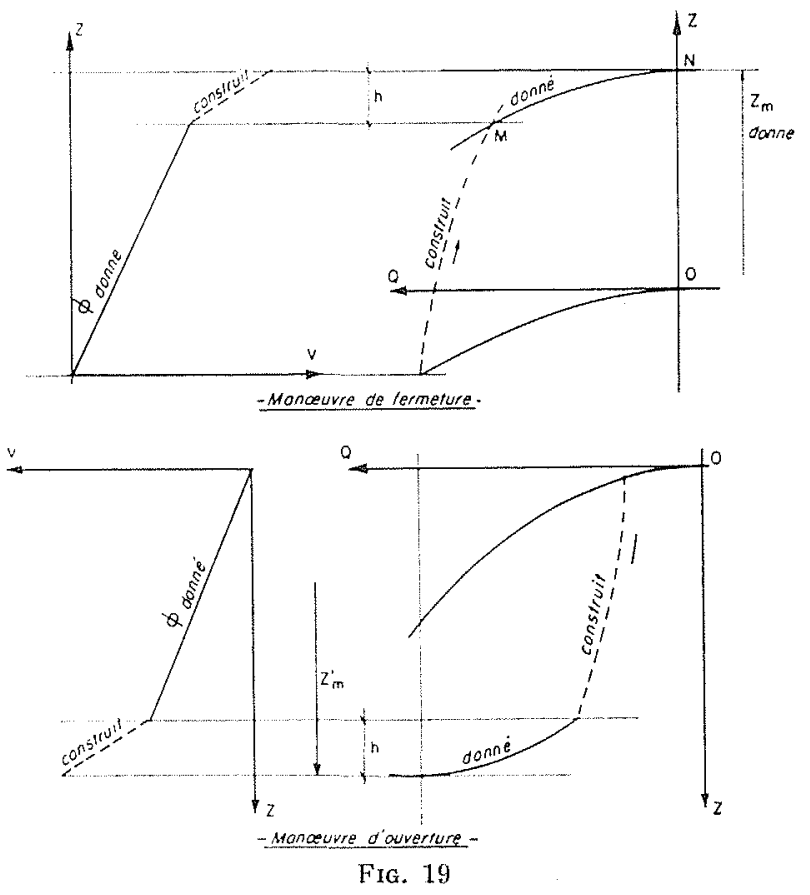

cylindrique se rapproche assez d'une parabole d'axe vertical ayant son sommet on N. Il suffira donc de tracer une telle parabole sur l'épure et de construire ensuite la courbe $Z$ (V) par la méthode directe. Cette courbe peu accentuée pent facilement ìtre assimilée à une droite dont l'inclinaison donnera la section de la chambre d'expansion que l'on cherche.

Un cas particulier intéressant est celui ou $\mathrm{H}_{\text {n }}$ reste constante au cours de la manœurre considérée. Ce cas peut se produire effectivement lorsque la parabole des pertes de charge est égale à la parabole MN. Dans ce cas, la combe des ro. lumes cherchée est une droite.

En effet, les equations du mouvement s'écrivent alors :

$\left\{\begin{array}{l}\frac{\mathrm{L}}{g f} \frac{d \mathrm{Q}}{d t}=Z_{v}+\mathrm{K}_{1}(\mathrm{Q})^{2} \\ \left.Z+\mathrm{K}_{1}(\mathrm{Q})^{2}\right)=Z_{j i}-\mathrm{A} \mathrm{Q}^{2}(\mathrm{~A}=\text { Cste relative } \dot{\mathrm{a}}\end{array}\right.$ la parabole $\mathrm{MN}$ ).

$\mathrm{Q}=-\mathrm{F} \frac{d \mathrm{Z}}{d t}$ 
Tous calculs faits, on trouve :

$$
\mathrm{F}=\frac{\mathrm{L}}{2 g f\left[Z_{m} \mathrm{~K}_{1}-\mathrm{Z}\left(\mathrm{K}_{1}-\mathrm{A}\right)\right]}
$$

qui correspond bien à une surface constante si $\mathrm{K}_{1}=\mathrm{A}$ (hauteur accélératrice constante).

Or, ce cas peut se présenter assez souvent dans la pratique, et de toutes façons, on conçoit assez bien que plus on se rapproche de la condition $\mathrm{H}_{a}=$ Cste, plus la courbe $Z(V)$ est voisine d'une droite.

\section{Calcul a l'ouverture}

Le problème se résoud exactement de la même manière (fig. 19).

c) Troisième probleme. - - Un cas sur lequel nous allons nous étendre plus longuement est le suivant :

Entre les deux types les plus courants, cheminée à étranglement optimum et cheminée à chambre d'expansion, il existe toute une gamme de cheminées d'un type intermédiaire avec étranglement inférieur et chambre d'expansion. Ce type de cheminée a, sur les deux types extrêmes, certains avantages, car il hérite des qualités de chacun des deux types. Prenons un exemple :

Soit un aménagement défini par les caractéristiques suivantes, $\mathrm{L}, f, \mathrm{Q}$, la galerie étant supposée déjà calée (ce qui est malheureusement souvent le cas), le fond de la cheminée est donné. On se donne de mème une surpression maximum $Z_{m}$ dans la galerie au droit de la cheminée d'équilibre. Il arrive souvent que, pour une telle installation, on ne puisse prendre les deux types extrêmes.

\section{Type à étranglement optimum}

- parce que le puits est trop important,

- parce que $R_{2}$ est trop fort et risque, en cas de déclenchement brutal, de détériorer la galerie (1),

- parce que les manouvres partielles ou lentes risquent d'entrainer une montée du niveau supérieure à celle de la fermeture totale instantanée.

(1) M. Yves Ponsar a mis en garde, dans un article patu dans le $\mathrm{I}^{\circ} 2-1950$ de la Houlle Blanche, les ingénieurs contre le danger d'un étranglement trop puissant, dans le cas de galeries trop longues. Cet étranglement produit, en effet, en cas de déclenchement rapide, une onde de surpression qui remonte le long de la galcrie jusqu'à la distance $\frac{a \mathrm{~T}}{2}$ de la prise d'eau $a$ étant la célérité des ondes et 'T le temps de fermeture du vannage).

\section{Type à chambre d'expansion}

-.- parce qu'à la moindre manouvre de vannage, le niveau de l'eau oscille des chambres supérieures aux chambres inférieures, ce qui est néfaste pour la régulation et surtout pour la galerie. On peut évidemment diminuer cet inconvénient en faisant un puits vertical plus large, mais c'est aux dépens d'une augmentation importante de volume.

Dans ce cas, le type intermédiaire s'impose; on se donnera, à priori :

- un étranglement $\mathrm{R}$ compatible avec la solidité de la galerie,

- un puits central d'un diamètre $\varnothing$, donné soit par la section de Thoma, soit par des raisons constructives, soit par toute autre raison particulière.

Les procédés directs ou indirects conjugués permettent de résoudre, en deux épures, le probleme :

$1^{\circ}$ Calcul à la fermeture totale (instantanée ou progressive). - Comme pour la cheminée à ćtranglement optimum, la cheminée scra calculée au mieux si, à partir du moment où l'eau en montant atteint le radier de la première chambre d'expansion, la pression sous l'étranglement reste constante et égale à $Z_{m}$ (donné).

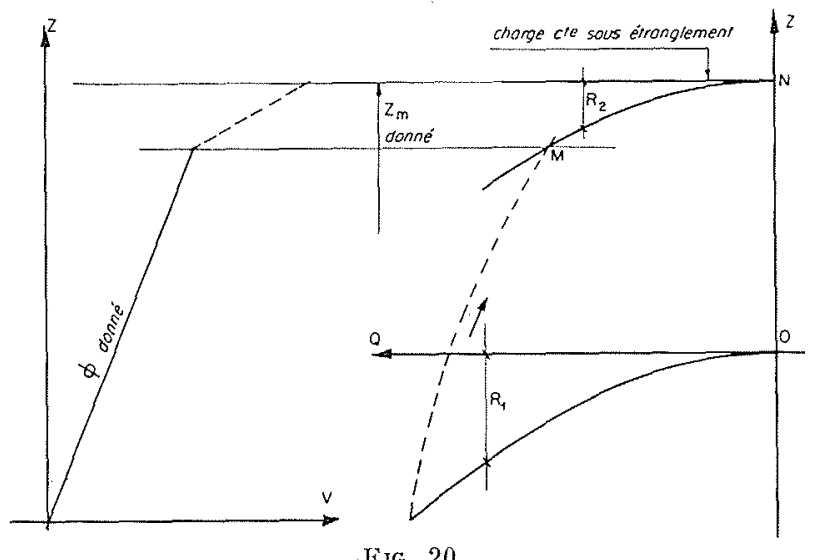

Cela revient, dans le graphique $Z(Q)$, à définir la surpression au cour's de la montée de l'eau dans la chambre d'expansion, par une droite horizontale de cote $Z_{m}$ (fig. 20); ce qui définit, en même temps, la courbe de la montée d'eau [parabole $R_{2}=K_{2}(Q)^{2}$ ].

Comme pour la cheminée à chambre d'expansion simple, lo bas de l'épure se construira par 


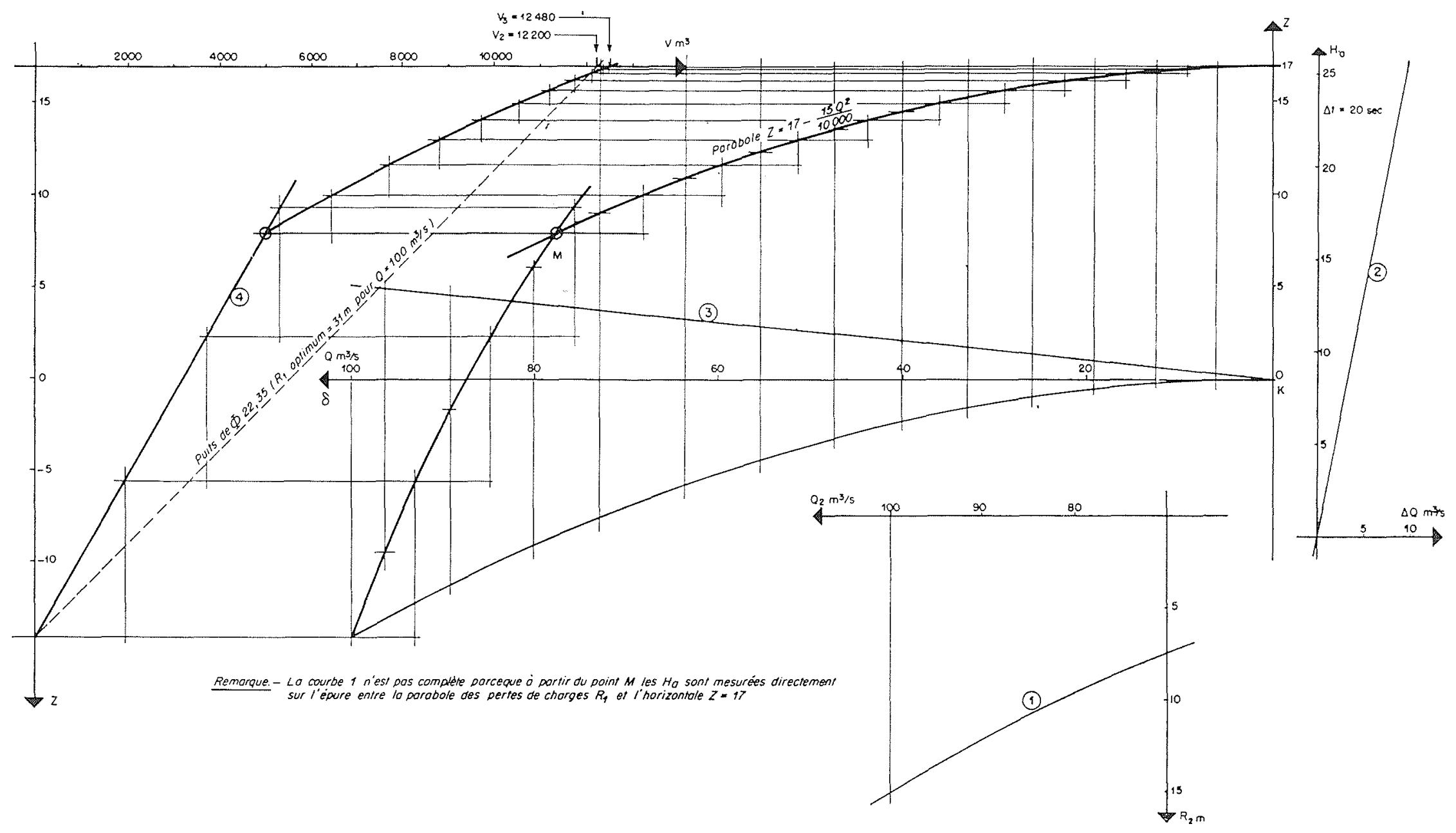

EPURE $N^{\circ} 4$ 
la méthode indirecte; la courbe $\mathrm{Z}=f(\mathrm{Q})$ obtenue coupera la parabole au point $M$. A partir de $\mathrm{M}$, on emploiera la méthode directe qui permettra de terminer la courbe des volumes jusqu'au point N. Le calcul analytique s'applique d'ailleurs également dans ce cas, à partir du point oì la pression reste constante.

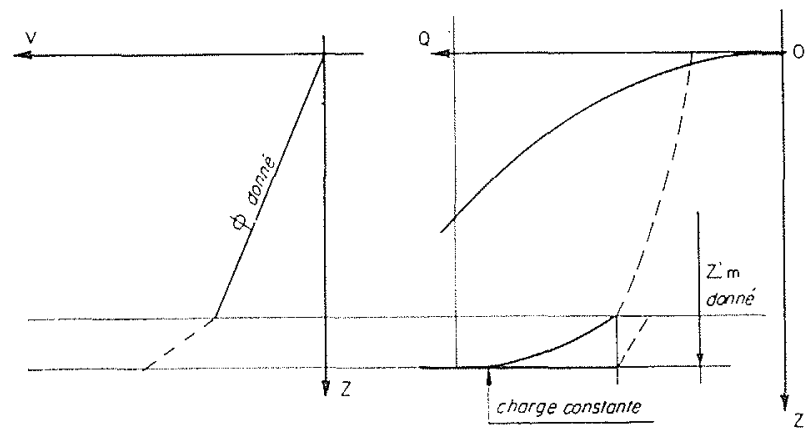

FxG. 21

$2^{\circ}$ Calcul à louverture. - On se donne, à priori, la condition d'ouverture la plus difficile à réaliser, par exemple ouverture de $x$ à $100 \%$ (progressive ou instantanée), ainsi que la valeur de l'étranglement si celui-ci est dissymétrique.

Comme dans le cas d'une fermeture, on construira l'épure par les méthodes indirecte et directe conjuguées (fig. 21).

\section{Remarque}

$1^{\circ}$ Ainsi que nous l'avons vu précédemment, la partie de la courbe des volumes que l'on construit est, en général, presque rectiligne. Pratiquement, il suffit de la remplacer par deux segments inserits dans la courbe pour se rappro-

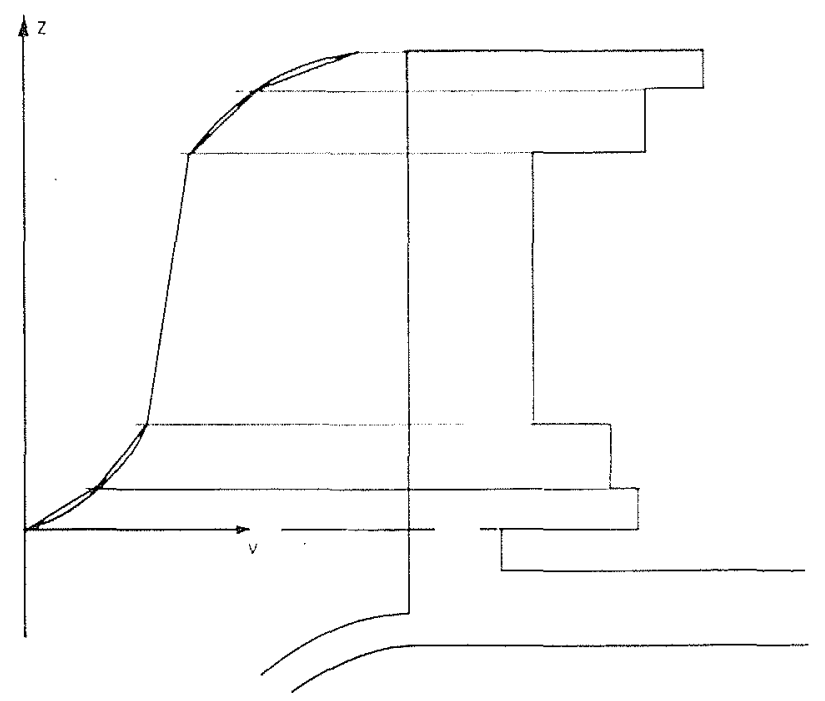

Fili, 22 cher presque parfaitement de la condition de pression constante.

Les chambres d'expansion, comme le montre la figure 22, ont une forme très réalisable en pratique. Il est d'ailleurs souvent commode, pour cette raison, de prévoir deux étages de chambres d'expansion superposées.

$2^{\circ}$ Une telle cheminée a, en général, à $Z_{j i}$ égal, un volume très voisin de celui de la cheminée d'équilibre à étranglement optimum; la différence de volume pouvant être à l'avantage de l'un ou l'autre type suivant le cas. De toute façon, si la cheminée à étranglement et chambre d'expansion est d'un volume légèrement supérieur, cette différence est largement compensée par la progressivité de la mise en charge de la galerie au cours d'un déclenchement ainsi que par sa forme souvent mieux appropriée aux travaux du Génie Civil.

\section{Application pratique (épure $n^{\circ}$ 4).}

Nous avons adapté à l'installation hydraulique de l'exemple $n^{\circ} 3$ une cheminée à étranglement et chambre d'expansion créant la même charge max. $Z_{m}$ sous l'étranglement. Nouns nous sommes ainsi donnés à priori :

- le diamètre du puits $\varnothing 17 \mathrm{~m}$,

- la valeur de l'étranglement $R_{2}=15 \mathrm{~m}$ pour $\mathrm{Q}=100$ (au lieu de $\mathrm{R}_{2}=31$ dans l'exemple $n^{\circ} 3$ ),

- la charge max. sous étranglement :

$$
Z_{n}=17 \mathrm{~m} \text {. }
$$

A l'aide d'une épure de fermeture totale instantanée, employant les procédés direct et indirect combinés, on cherche les dimensions des chambres d'expansion supérieures créant une charge sous étranglement constante et égale à $Z_{m}$ à partir du moment où l'eau atteint le radier des chambres d'expansion.

L'épure donne 9,10 $\mathrm{m}$ comme hauteur totale des chambres d'expansion. Dans la courbe des volumes, on mesure sur l'horizontale de cote $Z_{\mathrm{m}}=17$, le volume de la cheminée d'équilibre, on trouve :

$$
\mathrm{V}_{3}=12.480 \mathrm{~m}^{3}
$$

Comme on l'a vu, la courbe de volume théorique donnée par l'épure peut pratiquement être approchée de très près au moyen de deux étages de chambres d'expansion. Dans le cas parti- 
culier de l'épure, l'augmentation de volume qui en résulte est inférieure à $1 \%$.

Le volume de la cheminée cylindrique à étranglement optimum, de même $Z_{m}$ déjà donné dans l'exemple $n^{\circ} 3$, est :

$$
V_{2}=12.200 \mathrm{~m}^{3}
$$

La différence relative $\frac{V_{3}-V_{2}}{V_{3}}$ théoriquement égale à :

$$
\frac{12.480-12.200}{12.480}=2,2 \%
$$

est donc pratiquement de l'ordre de $3 \%$.

Ainsi, avec une forme appropriée et pour un même $Z_{m}$, on peut réduire l'étranglement $R_{2}$ de 31 à $15 \mathrm{~m}$ sans augmentation appréciable du volume de la cheminée. D'ailleurs, cette différence de $3 \%$ aurait pu être réduite à zéro, si on avait choisi un puits vertical de diamètre plus petit.

\section{CONCLUSION}

Toutes les fois qu'on s'oricntera vers une cheminée d'équilibre peu classique, ou à propos de laquelle se poseront des questions spéciales, les procédés rétrogrades et directs pourront être d'une grande utilité car ils permettront d'éviter de longs tâtonnements.

Le procédé direct permet, en particulier, un dosage judicieux entre l'insertion des chambres d'expansion et l'utilisation d'un étranglement. Ainsi, en disposant les chambres de facon convenable, on peut fort bien, tout en diminuant considérablement la force de l'étranglement, conserver le même volume que la cheminée cylindrique à étranglement optimum (de même montée maximum).

Enfin, même si la taille de l'ouvrage ne justifie pas l'adoption d'une solution de ce genre, l'emploi de ces procédés permettra souvent d'en trouver rapidement les dimensions approximatives.

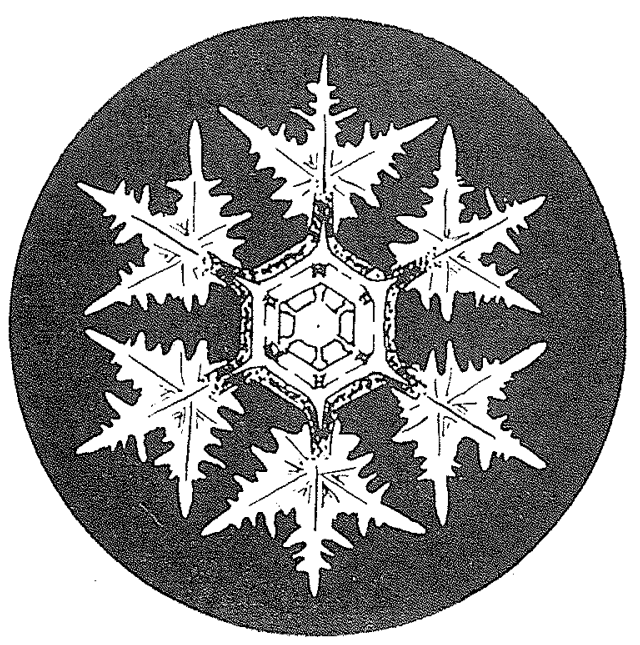




\section{A graphical method of analysing surge tank oscillations}

\section{INTRODUCTION}

The two articles * which are summarized below constitute an interesting series of applications of the graphical method of surge tank analysis originally devised by Schoklitsch (1) and enlarged on by his compatriot Schiffmann (2) and the Italian engineer Del Felice (3).

In using this method, the author's stress the importance of the new conception of the accelerating head, $\mathrm{H}_{\mathrm{n}}$, in the surge tank; another innovation which considerably enlarges the scope of the method is the use of a movable ax-axis for the study of gradual flow variations.

Apart from this, the interest of the articles is threefold :-

(i) They cover in a particularly clear way the more standard cases of surge tanks-plain tanks with or withont changes of cross section, overflow tanks and differential and throttling tanks.

(ii) Details are given of the procedure to adopt in making second order approximations at each successive step in the graphical analysis; this techrique, though familiar enough to those experienced in step-by-step calculations, has not hitherto been discussed in the literature on the Schoklitsch method.

(iii) Finally the method is applied to some quite complicated cases of practical occurrence, e.g. where several surge tanks are placed in series on the headrace tumnel. The articles conclude with what is undonbtedly an important contribution to surge-tank design, i.e., a method whereby one can directly compute optimum dimensions of these structures without making any preliminary trial computations.

\section{NOTATION}

The principal symbols used are as follows:

$\mathrm{F}=$ Surge tank cross section.

$\mathrm{f}=$ Cross section of head-race tunnel.

$\mathrm{L}:=$ Length of head-race tunnel.

$\mathrm{Q}_{2}, \mathrm{Q}_{1}, \mathrm{Q}_{3}=$ Discharges in the head-race tunnel, surge tank and penstock respectively, counted as positive when directed towards the foot of the tank.

$$
\mathrm{Q}=\mathrm{Q}_{1}=-\mathrm{Q}_{2}-\mathrm{Q}_{3} \text { (see below). }
$$

\footnotetext{
* See also la Houille Blanche, $n^{\circ}$ 5, 1950.
}

$(1,2,3)$ See list of references at end. $\mathrm{R}_{1}=\mathrm{K}_{\mathrm{1}}\left(\mathrm{Q}_{1}\right)^{2}$ is the head loss in the head race
lunnel.

$\mathrm{R}_{2}=\mathrm{K}_{2}\left(\mathrm{Q}_{2}\right)^{2}$ is the head loss at the inlet to the surge tank.

$\mathrm{Z}_{0}=$ Water level at intake (static head).

$Z_{\mathrm{r}}=$ Water level in the tank (variable).

$Z_{\mathrm{p}}=\mathrm{Z}_{0}-\mathrm{R}_{1}-\mathrm{R}_{2}$

$Z=Z_{\mathrm{v}}-Z_{0}$.

$\mathrm{H}_{\mathrm{\Omega}}=\mathrm{Z}_{\mathrm{v}}-\mathrm{Z}_{\mathrm{p}}$ is the so-called accelerating head

$\mathrm{V}=$ Volume of water in the surge tank.

$\mathrm{W}=$ Velocity of water in the head race tunnel (same sign convention as for $\mathrm{Q}_{1}$ ).

$\mathrm{s}=$ Acceleration due to gravity.

$\alpha=\frac{g}{\mathrm{~L}} \mathrm{f}$

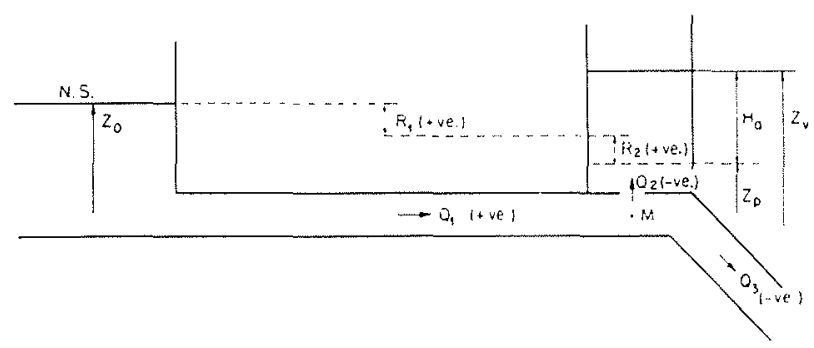

Fig. 1.

It is to be noted that $\mathrm{R}_{1}$ and $\mathrm{R}_{2}$ may be either positive or negative according to the direction of flow; in the present articles they are positive, if as indicated in the accompanying fighre.

Most of the quantities defined above vary in the course of time and to indicate which moment is being referred to, subscripts are added as follows : $Q_{1}, Z_{V(t+\Delta t)}$ and so on. In this connexion, a small interval of time is writlen as $\Delta t$, in the usual way; the distinction between the $\Delta$ and $\mathrm{d}$ signs to be found in the text is also standard-the first representing small but finite differences and the second, infinitesimals.

Finally, a dash is frequently used in the various graphs to denote minus quantities, positive ones being written as above (e.g. $\mathrm{Z}$ and $\mathrm{Z}^{\prime}, \mathrm{Q}$ and $\mathrm{Q}^{\prime}$, etc.) 


\section{Resume of the first article appearing in La Hounlle Blanche for September-Oetober, 1950}

We assume that readers can reler to the French lext as required.

\section{BASIC FORMULAE UNDERLYING THE GRAPHICAL. CONSTRUCTION (pp. 535-537)}

(i) Equation of conlinuily :

$$
Q_{1}+Q_{2}+Q_{3}=0
$$

(ii) Equation of motion :

Neglecting the acceleration of the water in the lank, we find that * :

$$
\mathrm{H}_{\mathrm{t}}=-\frac{\mathrm{L}}{\mathrm{g}} \mathrm{f} \frac{\mathrm{dW}}{\mathrm{dt}}
$$

$$
\text { Noll } \quad \text { dW }=\frac{\mathrm{d} Q_{1}}{\mathrm{f}}
$$

Moreover, if we assume for the moment that $\mathrm{Q}_{3}$ is constant, we have that

$$
\mathrm{d} Q_{1}+\mathrm{d} Q_{2}=0
$$

and thence finally that *

$$
\begin{aligned}
\Delta Q & =\Delta Q_{1}=-\Delta Q_{2} \\
& =-\alpha H_{: t} \Delta t
\end{aligned}
$$

(iii) Finite difference equations derived from the foregoing: For the discharge we find that

$$
Q_{2(t+\Delta i)}=Q_{21}+\Delta Q
$$

where *

$$
\Delta Q=-\alpha H_{a t} \Delta t
$$

and for the water level in the lank, that

$$
Z_{\mathrm{v}(\mathrm{t}+\Delta \mathrm{t})}=Z_{\mathrm{vt}}+\Delta \mathrm{Z}
$$

where *

$$
\Delta Z=-\frac{Q_{2 t}}{F} \Delta t
$$

* The equations marked with an asterisk are incorrectly stated in the original text; in the present summary however they have been rewritten in the correct form.
THE GRAPHICAL, CONSTRUCTION (pp. 537-543)

The method consists essentially in a graphical procedure for simultaneously solving equations (1) and (2); no doubt one of the longest operations required is the preparation of the various preliminary curves (at least 5 in number) needed for the construction of the graphical solution properly speaking. In passing it should be pointed out that Messrs. Bouvard and Monbert have made a notable improvement on the method as originally given by the substitution of $\mathrm{Q}_{1}$ for $\mathrm{W}$ as the $\mathrm{x}$-coordinate of the main graph (see fig. 3).

A) Construction of preliminary curves

(fig. 3, p. 538) :

(i) Preliminary curve $\mathrm{N}^{\circ} 1$ :

$\mathrm{R}_{2}$ is first plotted against $\mathrm{Q}_{2}$ in the normal way (i.e., $\mathrm{Q}_{2}$ positive towards the right).

(ii) Preliminary curve $\mathrm{N}^{\circ} 2$ :

On another sheet is traced the straight line given by equation (1), with $\mathrm{H}_{\mathrm{a}}$ as the $\mathrm{y}$-coordinate, with $\mathrm{Q}$ plotted backwards as the $\mathrm{x}$-coordinate and for $a$ suitably chosen time interval $\Delta \mathrm{t}$.

(iii) Main graph; preliminary curve $N^{\circ} 3$ (fig. 3, diagram in centre) :

It is on this graph that the construction of the graphical solution will ultimately be carried out. For this purpose the following coordinates are chosen :

$\mathrm{x}$ axis.... $\mathrm{Q}_{\mathrm{I}}(\mathrm{Q})$, with positive values towards the left (i.e., backwards).

y $\operatorname{axis} \ldots \mathrm{Z}=\mathrm{Z}_{\mathrm{y}}-\mathrm{Z}_{0}$.

The scales chosen for these variables are the same as those alreatly chosen for the two foregoing graphs.

However, on the same diagram are drawn two other preliminarly curves and a pair of subsidiary axes, defined as follows :

(a) The lower curve in the diagram (unnumbered) gives $\mathrm{R}_{1}$ as a function of $\mathrm{Q}$.

(b) Curve (3) represents the volume relationship.

$$
\Delta \mathrm{V}=\mathrm{Q} \Delta \mathrm{t}
$$

This curve requires the definition of a suitable volume scale against the $\mathrm{y}$ axis, and like (a), is drawn to pass through the origin 0 of the main axes.

(c) The subsidiary axes $\hat{\delta} \hat{o}-\alpha \alpha$ :-

Firstly, the ax axis is in the nature of a graphical representation of the continuity equation

$$
Q_{1}+Q_{2}+Q_{3}=0
$$


Hence, suppose that we are given the value of $\mathrm{Q}_{3}$, which we will here presume negative; set off this value of $Q_{3}$ along the $\mathrm{QQ}^{\prime}$ axis in the usnal waty towards the left, and draw in the ar axis as shown in fig. 4. $\mathrm{Q}_{2}$ may then be found for any point such as A by measuring its distance in the normal way (positive values towards the right) from the a.e axis. The construction is shown in fig. 4 of the text for + ve. $Q_{1}$ and - ve. $Q_{2}$ and $Q_{3}$

The ố axis is found by drawing a horizontal straight line through the intersection of line (3) and the axis $\alpha \alpha$.

(iv) Preliminary curve $\mathrm{N}^{\circ} 4$ :

This gives the relationship between the volume $V$ and the height $Z_{x}$ in the tank; notice however that the quantity $\mathrm{V}$ is plotted along the $\mathrm{x}$ axis instead of along the $y$ axis as is usual. The scales chosen are the same as those used in ploting curve 3.

(v) Preliminary curve $\mathrm{N}^{\circ} 5$ :

This curve is required should the surge tank be fitted with an overflow, and gives the discharge passing over the weir in time $\Delta t$ as a function of the level $Z_{v}$ in the tank (in actual fact, it is the inverse relation which is plotted, just as for curve 4).

B) STEP-BY-STEP CONSTRUCTION (1 st. approximation) (figs. $5 \& 6$ ).

Suppose A (figs. 3, 4 5 ) is the point denoting the level and discharge in the tank at the instant $t$; it is required to locate the point $B$ giving the level and discharge at the instant $(\mathrm{t}+\Delta \mathrm{t})$.

The steps in the construction are as follows:

(i) $\mathrm{H}_{a}$ is first found for the initial instant by setting off $\mathrm{R}_{1}$ plus $\mathrm{R}_{2}$ below the $\mathrm{Q}^{\prime}$ axis (as in figs. ' or 4) and measuring down from $A$.

(ii) $\Delta Q$ can then be immediately found by scaling off on curve 2, as shown in fig. 5. This quantity is then immediately set off to the left or right of point $\mathrm{A}$, according to its sign, as shown in the same figure.

(iii) Referring now to figure 6 of the text, it will be readily seen that the increment of volume $\Delta \mathrm{V}$ of water in the tank during the interval $\Delta t$ is given (algebraically) by the intercept cd. This intercept is now set off from the point e on curve 4, as shown on the left, and enables the point $\mathrm{g}$ to be picked off on the same curve.

(iv) We now have (figs. 5 \& 6 respectively) two lines drawn parallel to the axes bearing the title "lieu de B". The intersection of these two lines (which are of course really drawn on the same diagram) then gives the point $\mathrm{B}$ as required.

C) STEP-BY-STEP CONSTRUCHION (2nd. approximation) (figs. 9, 10 \& 11)

In paragraph B the various quantities such as $\mathrm{H}_{\mathrm{a}}$ which entered into the step-by-step calculations were supposed not to vary during the time interval $\Delta \mathrm{t}$; in other words, we used their initial values to calculate the rise in level and the change in discharge occurring throughout this interval.

It is therefore most aesirable to repeat the calculations using the mean values of $\mathrm{H}_{\Omega}$ etc., which have been determined from the first approximation; the author's give a number of practical tips on the best way of doing this (as for instance, how to extrapolate from the part of the curve already drawn), but these will not be treated in detail here.

\section{D) Remariks on the graphical CONSTRUCTION}

\section{(i) Choice of $\Delta t$ :}

The value of $\Delta \mathrm{t}$ is usually fixed at 5,10 or 20 seconds; the final choice depends partly on the degree of accuracy required, and partly on the natural period of oscillation of the surge tank and head race tunnel combined.

(ii) Gradual opening or closure of the turbines : Use of the movable $\alpha x$ axis.

A fixed position of the axaxis corresponds to a fixed value of the discharge $\mathrm{Q}_{3}$, and hence in reality to an instantaneous opening or closure of the turbines (the acs axis is brought to its new position right at the start of the surge, at $\mathrm{t}=0$, and is not varied thereafter).

In order to analyse non-instantaneous movements it is evidently necessary to bring this axis to its final position in a number of steps; examples of this procedure, for linear opening and closing movements respectively, are given in Epures $N^{\circ} 2$ and 3 of the French text.

(iii) Rise in the surge tank as a function of time :

The $Z_{\mathrm{v}}-t$ curve may be easily deduced from the solution curve as found above, seeing that for each point of the latter we know both the level $\mathrm{Z}_{\mathrm{v}}$ and the lime $\mathrm{t}$.

\section{APPLICATIONS OF THE GRAPHICAL METHOD TO DHFFERENT PRACTICAL CASES}

\author{
(page 543 to the end).
}

(i) Simple tank, of variable cross-section :

The procedure is exactly as described above; starting from an initial point such as A the operational curve is constructed step by step.

See p. 543, "Epure $N^{\circ} 1$ " for a practical example of such a case-that of the Surge Tank of the Passy Power Plant.

(ii) Tank fitted with overflow :

The procedure is as in (i) until the water in the tank reaches the weir crest. We then have to switch from curve 4 to curve 5 (see fig. 3 ) in order to determine the subsequent rise of level in the surge chamber.

A numerical case has been worked out by the authors (see "C", page 546, and" Epure $N^{\circ} 3 "$ ").

(iii) Throttling tank :

No extra complication is introduced if, as is frequenlly the case, the stand pipe is fitted with a throttling intake; the head loss $\mathrm{R}_{2}$ must of course be suitably chosen. 
The authors were called upon to study an actual installation of this type for the Randens Power Station; one of their results is given by the "Eplure $N^{\circ} 2$ " on page 54h (for the initial conditions see under $\mathrm{B}$ on page 546 ).

(iv) Differential surge tank :

Some extra complications are introduced, as the struclure is now composed of two stand pipes, the discharges in which satisfy the following relation :

$$
\mathrm{Q}_{2}^{\prime}+\mathrm{Q}_{2}^{\prime \prime}=\mathrm{Q}_{2}
$$

and one of which is fitted with a throttling intake. In addition, the stand pipe which is not throltled is provided with an overflow.

The procedure to adopt in this case is illustrated by figs. 7 \& $8 ; \mathrm{A}^{\prime \prime} \mathrm{A}^{\prime}$ is equal to the head loss due to the throttling intake, and is found from curve 1. This latter curve is drawn in such a way as to facilitate the determination of $\mathrm{Q}_{2}{ }^{\prime}$ and $\mathrm{Q}_{2}{ }^{\prime \prime}$, and of the corresponding $\mathrm{V}^{\prime} \mathrm{s}$. The rest of the construction presents no special difficulty, the graphical solution being represented by the curves marked $\mathrm{c}^{\prime}$ and $\mathrm{c}^{\prime \prime}$ in the two figures mentioned.

An actual example of such an installation is that treated under the heading D on page 546 ("Epure $N^{\circ} 4$ ").

(v) Surge tank located on a main head-race tunnel downstream from a bifurcation (see fig. 13).

We need at the outset a few additional symbols :-

$Q_{1}$, Discharge in the main tunnel.

$\mathrm{q}^{\prime}, \mathrm{q}^{\prime \prime}$, Discharges in feeder tunnels, positive if as in fig. 13.

$\mathrm{f}^{\prime}, \mathrm{f}^{\prime \prime}, \mathrm{f}$, Cross-sections of tunnels.

$\mathrm{z}_{0}{ }^{\prime}, \mathrm{z}_{0}{ }^{\prime \prime}$, Water levels at intakes to feeder lunnels.

$\mathrm{r}^{\prime}, \mathrm{r}^{\prime \prime}$, Head losses in feeders.

$\mathrm{R}_{1}$,Head loss in main tunnel.

To solve this type of problem, the authors make use of a fictitious piezometric tube located at the triple point, i.e., junction of the three tunnels. This tube, though of infinitesimal cross-section, can then be regarded as a subsidiary surge tank, and in this way the problem can be reduced to that of a series of surge tanks of simple type for which the graphical method of solution has already been fully discussed.

We then have the following equations * :

$$
\Delta \mathrm{q}^{\prime}=-\frac{\mathrm{gf}{ }^{\prime}}{\mathrm{I}^{\prime}} \mathrm{h}_{\mathrm{a}}{ }^{\prime} \Delta \mathrm{t}=-\mathrm{k}^{\prime} \mathrm{h}_{\mathrm{a}}{ }^{\prime} \Delta \mathrm{t},
$$

say, a similar equation for the second feeder tunnel, and *

$$
\Delta Q_{1}=-\frac{\mathrm{gf}}{\mathrm{I}} \mathrm{H}_{\mathrm{a}} \Delta \mathrm{t}=-\mathrm{KH}_{\mathrm{a}} \Delta \mathrm{t}
$$

for the main tunnel.

There are moreover two continuity equations :

$$
\begin{gathered}
Q_{1}=q^{\prime}+q^{\prime \prime} \\
\Delta Q_{1}=\Delta q^{\prime}+\Delta q^{\prime \prime}
\end{gathered}
$$

(a) Preparatory remarks :

The frame of reference chosen for the main graph. is similar to that chosen above-we plot $Z_{\mathrm{v}}$ vertically and $Q$ (i.e., $Q_{1}$ ) backwards horizontally. A snag is evidently introduced by the fact that there is no single static head, such as $Z_{0}$ previonsly, to which the $\mathrm{Z}$ scale can be referred; this is overcome by quoting $Z_{\mathrm{x}}$ in terms of R.L. (see Epure $N^{\circ} 5$ for example) and by drawing the head loss curve for the main tunnel to pass through any convenient point on this scale. On the same fign'e are also drawn the head loss curves for the feeder tunnels, cutting the $\mathrm{Z}$ axis at the corresponding static R.L.'s for each intake.

For simplicily, only the case of a non-throttling surge tank is studied by the authors, though no extra complication is really added to the construction by a throttling intake.

At a given instant $\mathrm{t}$, suppose that the values of :

$$
\mathrm{q}^{\prime}, \mathrm{q}^{\prime \prime}, Q_{1} \text {, and of } Z_{\mathrm{v}}
$$

are all known.

By means of a graphical construction, we shall determine the value of the pressure at the triple point at the same instant $\mathrm{t}$, and the values (to a first approximation) of the variables given above at time $(\mathrm{t}+\Delta \mathrm{t})$. In order to do this, we shall provisionally assume that the pressure at the triple point is known at the initial instant, as represented by point $\mathrm{P}$ in fig. 15.

(b) The graphical construction :

(i) To find the accelerating head for the main tunnel, the head loss $\mathrm{R}_{1}$ is sublracted graphically from the pressure $\mathrm{P}$ at the triple point, giving point $\mathrm{E}$. If $\mathrm{A}$ is the point representing the level in the surge chamber at the same instant $\mathrm{t}$, then the height AE gives the required accelerating head. As a malter of convenience it is however preferable to set off a line from $\mathrm{A}$, equal to $\mathrm{R}_{1}$ in length, in the opposite direction (see fig. 15), giving point $\mathrm{B} ; \mathrm{BP}$ is then equal to the accelerating head.

(ii) From $\mathrm{B}$ a straight line making an angle of tangent $\mathrm{K \Delta t}$ with the vertical is drawn; the lenglh of the intercept made by this line with a horizontal line passing through $\mathrm{P}$ gives the change in discharge occurring in the main tunnel during the time interval $\Delta \mathrm{t}$.

(iii) For the leeder tunnels the construction is similar : the accelerating heads are first found (remember that $\mathrm{P}$ now plays the role of $\mathrm{A}$ in the preceding discussion), and intercepts corresponding to $\Delta \mathrm{q}^{\prime}$ and $\Delta \mathrm{q}^{\prime \prime}$ found as before by inter'section (see fig. 16 of the French text).

(iv) As already explained, we do not really know the position of $\mathrm{P}$ to begin with, but it must in fact be found from the continuity equation, as follows :-

Seeing that we know the values of $\mathrm{q}^{\prime}$ and $\mathrm{q}^{\prime \prime}$ at time $t$, we can locate points $\mathrm{i}^{\prime}$ and $\mathrm{i}^{\prime \prime}$ on the vertical drawn through point $\mathrm{A}$, as shown in fig. 17. From points $\mathrm{B}, \mathrm{i}^{\prime}$ and $\mathrm{i}^{\prime \prime}$ are drawn lines making the same angles with the vertical as above (artan $\mathrm{K} \Delta \mathrm{t}$, artan 
$\mathrm{k}^{\prime} \Delta \mathrm{t}$ and artan $\mathrm{k}^{\prime \prime} \Delta \mathrm{t}$ respeclively). The intersection of the first with the sum of the two others then gives the ordinate of $\mathrm{P}$, as required. The proof of this construction is almost self-evident, once it is realised that the heights i'P and $\mathrm{i}^{\prime \prime} \mathrm{P}$ give the proper acceleraling heads for the two feeder tunnels.

(v) To find the rise of level in the surge lank during the time interval $\Delta t$, we need only apply the fundamental equation

$$
\Delta Z=-\frac{Q_{2}}{F} \Delta t
$$

in the same way as before. It is once again necessary, of course, to plot curves 3 and 4 .

(vi) In accurate work it is good policy to carry out a second approximation in the same way as before, substituting mean values of the accelerating heads for the inilial values which were perforce adopted in the first calculations.

(c) Practical examples :

The authors give a practical example of an installation of the type considered above-that of the Passy Power Plant with the inclusion of the subsidiary intake on the Diosaz (see Epare $N^{\circ} 5$ in the
French text; the case dealt with is that of a tolul shut-down).

(vi) Surge tank located at the junction of two headrace tunnels :

This problem can be thought of as a special case of problem (v); however, the continuity equation is no longer required and the hypothetical piezometric tube is replaced by the surge chamber itself. In passing, the authors discuss two other methods which have been devised for installalions of this type :-

(a) GouTkin (4) has proposed an approximate method of analysis in which the two head-race tunnels are replaced by a single equivalent one; CALAME and GADEN's (5) curves can then be used in order to determine the amplitude of the oscillations for any given operation of the turbines. The method is however not exact.

(b) Escande (6) has studied the problem by numerical integration, using finite differences; the method is however ledious, can be applied to simple cases only and is cumbersome in the case of multiplesection structures.

The article concludes with a practical application of the author's' method to a ficlitious plant resembling the Bioge Power Station, the case treated being that of a linear closing operation lasting to seconds.

\section{Resume of the second article appearing in the present issue}

The present article forms a continuation of the first, and shows how the graphical method may be applied to a number of highly specialized, but important, problems. In particular, the authors have evolved a procedare (or, more exactly, a series of procedures adapted to various particalar problems) whereby the optimum dimensions of a surge tank can be found automatically, without the need of any preliminary trial analyses.

\section{I. - OTHER APPLICATIONS OF THE GRAPHICAI, METHOD}

\section{A) SEvEral SURge TANKS PLACED IN SERIES ALONG} THE ONE HEAD-RACE TUNNEL

This case is illustrated in fig. 1 of the French text. The notation is similar to that already used, subscripts being used to indicate the particular surge tank or section of head-race tunnel, etc., that is being referred to.

The principle anderlying the construction is simple; the head-race is broken up into a number of units, each consisting of the following components (working from downstream to upstream) :

(i) A surge chamber (say, $\mathrm{C}_{\mathrm{m}}$ ),

(ii) A section of head-race Innnel from $\mathrm{C}_{\mathrm{m}-1}$ to $\mathrm{C}_{\mathrm{m}}$, say),

(iii) The preceding surge chamber $\left(\mathrm{C}_{\mathrm{m}-1}\right)$ which plays the role of the intake in the simple cases discussed in the earlier article as far as the graphical computations are concerned.

Knowing the state of the system for time t, we froceed as before to find the state at time $t+\Delta t$; once again a second approximation must be made at each step in the construction. Part of a lypical solution in shown in fig. 4 , and, as will be seen, does not differ basically, from the simple case shown in fig. 3 of the first article.

Several practical comments should however be made :-

(i) It is generally rather difficult to start the graphical construction off. It may prove necessary to make repeated approximations in the very first $\Delta \mathrm{t}$ interval, and even to draw the first portion of the graphical solution to a larger scale than the rest.

(ii) Particular case of two surge tanks, one of which is considerably larger than the other :-

In this case it is legitimate to suppose that the smaller structure will have a negligible effect on the oscillations in the larger one. The operational curve can therefore be plotted for the latter on the supposition that the smaller tank is inexistant, and the oscillations in the smaller one may be derived subsequently from the first result. This approach proves particularly handy when a second approximation is made at each step in the solution; if a first approximation is considered good enough, nothing is in fact 
gained as the double solution may lhen be plotled directly.

The author's have gone to considerable pains in studying this lype of problem. The results of their sludy are shown in "Epure $N^{\circ} 1$ " of the French text, and represents a graphical solution of a trial problem where a small subsidiary shaft of 6 metres diameter is supposedly situated $4 \mathrm{~km}$. upstream along the head-race tunnel from the surge tank proper. The exact solution, found by making a second approximation at each step, is shown by the fall curve; dotted lines show the solution found by the first approximation only, and by the simplified approach described above, respectively. The first approximation turns out to be quite inexact, and in order to arrive at a reasonably accurate result it would be donbtless necessary to adopl a time interval $\Delta \mathrm{t} 5$ or 10 smaller. than that actually used; this would of contse make the graphical construction exceedingly intricate. The simplified approach is fairly good, though it is naturally rather difficult to judge at what stage the bigger structure will begin to be noticeably affected by the smaller.

B) Case in which the disgharge passing through THE TURBINES VARIES WITH THE HEAD.

Should the amplitude of the surges produced in the surge tank located at the entry to the penstocks (e.g., in the tank denoted as $\mathrm{C}_{\mathrm{n}}$ in the preceding discussion where there were n such structures in all - see fig. 5 of the French text): the discharge $Q_{(\mathrm{n}+1)}$ passing into the turbines may vary considerably while the oscillations are taking place, even should the power input be maintained rigorously constant during this time. Without going into a detailed description of the various cases which may crop up, which depend on the degree of interconnexion of the distribution network and so on, it is clear that the relation between $Q_{(\mathrm{n} * 1)}$ and the head can always be plotted on the functional graph of the surge tank oscillations as a curve $\Gamma$ (see fig. 5).

The graphical construction is scarcely more complicated than before, thanks to the use of the moveable ax axis. Figure 5 indicates the procedure to be followed : at an instant of time $t$, the as axis passes through a point $\mathrm{M}$ of the curve $\mathrm{\Gamma}$ of ordinate $Z_{n+1}$ equal to the head at the entry to the turbines. The latter is found from $\mathrm{Z}_{\mathrm{n}}$, the level of the water in the surge chamber, by adding (or subtracting, as the case may be) the head losses in the penstocks and at the surge tank inlet.

\section{II. - AUTOMATIC DESIGN OF SURGE TANKS}

In the present section of their article, the author's confine themselves to sludying simple layouts, as illustraled in fig. 6 of their text a single intake connected by a single head-race tunnel to the surge

* Represent an appreciable fraction of the total head.
Lank). The method can however be applied equally well to more complicated installations.

Before embarling on the delailed sludy of the various methods of automatic design which are available, it is worthwhile restating the basic equations governing surge tank oscillations. These, it will recalled, are as follows :

$$
\begin{gathered}
-\frac{\mathrm{L}}{\mathrm{gf}} \frac{\mathrm{dQ}_{1}}{\mathrm{dt}}=\mathrm{Z}_{0} \pm \mathrm{K}_{1}\left(\mathrm{Q}_{1}\right)^{2} \pm \mathrm{K}_{2}\left(\mathrm{Q}_{2}\right)^{2} \\
\mathrm{Q}_{1}+\mathrm{Q}_{2}=\mathrm{f}(\mathrm{t}, \mathrm{Z})
\end{gathered}
$$

where here of course, $\mathrm{f}$ is the usual functional symbol.

$$
Q_{2}=-F \frac{d Z}{d t}
$$

In writing these expressions we have eliminated $Q_{3}$. It will be remembered that the values of $\mathrm{L}$, $\mathrm{f}$ and $\mathrm{K}_{1}$ are known for a given project, and that $\mathrm{K}_{2}$ may be regarded as constant. The second equation enables gradual variations of discharge (and variations of discharge with the head) to be taken into account.

While flows are varying, $\mathrm{Q}_{1}, \mathrm{Q}_{2}, \mathrm{~F}$ and $\mathrm{Z}$ can be thought of as functions of t. However, since we have three equations only at our disposal, a fourth condition must be added in order to make the problem determinate. It is usual to fix on the form of the surge chamber, and hence $\mathrm{F}$ is known in terms of $\mathrm{Z}$; the trial design is then checked by calculation of the maximum and minimum levels reached during oscillations. This procedure is however by no means the only possible one, as the authors now show.

\section{A) Retrograde Method oF DESIGN}

\section{(i) Outline :}

This method simply consists in working backwards from a maximum or minimum level in the surge structure to find the permanent level existing in the struclure before the surge took place.

(ii) Graphical construction :

The graphical construction is similar to that described before, but is carried out in the opposite sense (that of decreasing $\mathfrak{t}$ ).

Suppose for example that we wish to determine the partial discharge of $\mathrm{x} \%$ starting from which an instantaneous increase of load up to $100 \%$ gives rise to an oscillation reaching a given level denoted by point $\mathrm{B}$ in fig. 7 . Ordinarily, $\mathrm{x}$ is given and the resulting fall in level found by the graphical construction, starting from point $\mathrm{A}$. The construction is however exactly similar if we set out from point $\mathrm{B}$ and work backwards in order to find point $\mathrm{A}$ as the intersection of the head-race tunnel head loss parabola with the curve $\mathrm{AB}$.

It should be remarked in conclusion that the retrograde method is only applicable to instantaneous changes of load; it would be doublless possible to handle non-instantaneous changes of regime by a method of successive approximations, but this would rob the method of its main advan- 
layt-which is precisely the avoidance of such stepby-step approximations.

\section{B) THE DIRECT METHOD OF AUTOMATIC DESIGN}

\section{(i) Outline :}

In the introduction to this section, it was pointed ont that a fourlh relationship has to be added to the the e basic equations of motion in order to make it possible to calculate surge tank oscillations. Instead of simply defining the cross-sectional area of the surge tank as a function of level, as is normally done, any other relationship between the variables could be conceivably chosen; while it is true that the majority of such choices are only of academic: interest, there are at least some which are definitely valuable. If in fact it were possible to design a surge tank so as to keep to prescribed variations of base pressure $\left(\mathrm{Z}_{\mathrm{y}}+\mathrm{K}_{\mathrm{2}} \mathrm{Q}^{2}\right)$ or of lvater level $\mathrm{Z}$ during the oscillations which occur in the tank after a sudden change of load, the surge tank problem would be solved in a most logical way. It is this method of design which the althors lerm "direct", as the structure is designed direclly to fulfil certain operailonal requirements.

Before describing the graphical procedure, it is worth noting that certain special problems can be solved algebraically, as follows :-

Suppose that it is desired to construct a surge tank such that the (back) pressure in the head-race tannel beneath the surge structure remains constanl dnring the first rise in level occurling after a complete shut-down.

The basic equations now become :-

$$
\begin{aligned}
\frac{L}{\mathrm{gf}} \frac{\mathrm{dQ}}{\mathrm{dt}} & =Z+K_{1}(Q)^{2}+K_{2}(Q)= \\
Q & =\mathrm{F} \frac{\mathrm{d} Z}{\mathrm{dt}}
\end{aligned}
$$

where $\mathrm{Q}$ denotes the discharge in the head-race tunnel $(+$ ve. towards the penstocks).

The functional requirement is of the form :

$$
\mathrm{Z}+\mathrm{K}_{2}(\mathrm{Q})^{2}=\text { Const. }=\mathrm{Z}_{\mathrm{m}}, \text { say. }
$$

If loe pul

$$
\frac{\mathrm{K}_{1}}{\mathrm{Z}_{\mathrm{m}}} \mathrm{Q}^{2}=\mathrm{q}^{2}
$$

and

lve find that

$$
\tau=\frac{L}{g f} \cdot \frac{1}{\sqrt{K_{1} Z_{m}}}
$$

$$
\frac{d q}{1+q^{2}}=-\frac{d t}{\tau}
$$

Hence

$$
\operatorname{artan} \varphi_{1} \operatorname{artan} \tau_{0}=\frac{t}{\tau}
$$

where go denoles the initial value of q.
It follows at once that

$$
\mathrm{Q}=\frac{\mathrm{Q}_{0} \sqrt{\mathrm{K}_{1} Z_{\mathrm{m}}}-Z_{\mathrm{m}} \tan \frac{\mathrm{t}}{\tau}}{\mathrm{K}_{1} \mathrm{Q}_{0} \tan \frac{\mathrm{t}}{\tau}+\sqrt{\mathrm{K}_{1} \mathrm{Z}_{\mathrm{m}}}}
$$

The second basic equation gives the surface of the surge chamber as a function of $\mathrm{Z}$ :

$$
\mathrm{F}=\frac{\mathrm{L}}{2 \mathrm{gf}\left[\mathrm{K}_{2} Z_{\mathrm{m}}+\mathrm{K}_{1}\left(Z_{\mathrm{m}}-\mathrm{Z}\right)\right]}
$$

that is, a rectangular hyperboloid.

The total volume of the structure may be found by integrating the expression for $\mathrm{F} . \mathrm{d} Z$ between the limits * $\mathrm{R}_{1}$ and $+\mathrm{Z}_{\mathrm{m}}$, and is equal to

$$
\mathrm{v} \frac{W^{2}}{2 \mathrm{~g}} \frac{1}{\mathrm{R}_{1}} \log _{\mathrm{e}} \frac{\mathrm{K}_{2} Z_{\mathrm{m}}+\mathrm{K}_{1}\left(\mathrm{Z}_{\mathrm{m}}+\mathrm{R}_{1}\right)}{\mathrm{K}_{2} \mathrm{Z}_{\mathrm{m}}}
$$

where $\mathrm{v}$ denotes the volume of the head-race tunnel. Seeing that * $Z_{\mathrm{m}}+\mathrm{R}_{1}=\mathrm{R}_{2}$, this formula reduces

finally to the following :

$$
v \frac{W^{2}}{2 g} \frac{1}{R_{1}} \log _{0}\left(1+\frac{R_{1}}{Z_{m}}\right)
$$

The importance of this expression lies in the fact that it gives the minimum volume of surge chamber required for a given pressure at the base of the structure; it moreover enables an estimate to be made of the variation of this minimum volume with change in base pressure. In passing, it should be noticed that for $\mathrm{K}_{1}=0$ the formula gives a chamber of constant section which, as is well linown, gives a theoretically constant base pressure in this particular case.

Though applicable in the most simple case, the analytical method of approach can scarcely be expected to succeed in more complicated problems, and recourse must then be taken to the graphical method. As we shall shortly see, the desired variation of base pressure may be readily traced on the usual $\mathrm{Q}-\mathrm{Z}$. graph, and the structure at once designed to fulfil this requirement (we can then construct the $Z-\mathrm{V}$ curve by the familiar step-by-step process).

(ii) Graphical construction :

As already mentioned, we suppose known

(i) The dimensions and head loss in the head-race tunnel.

* In hese equations, $R_{1}$ and $R_{2}$ denote the hendlosses in the head-race tunnel and in the throttling intake to the surge tank respectively corresponding to the maximum discharge of the turbines, and thus clifer in meaning from the same symbols as generally employed in the text. 
(ii) T'he curve $Z=\mathrm{f}(\mathrm{Q})$.

On this basis, we can plot the curve $Z=f(V)$ giving the volnme of the surge tank as a function of height.

(a) First approximation (see fig. $S$ of the French texi) :

Assume that the construction has been completed as far as the point $\beta_{\mathrm{t}}$ of the $\%-\mathrm{V}$ curve, the corresponding point on the $Z$ - - Q curve being denoted by $\mathrm{A}_{\mathrm{t}}$. For a given time interval $\Delta \mathrm{t}$, we wish to determine, firstly, the point $\mathrm{B}_{(\mathrm{t}+\Delta \mathrm{t})}$ on the $\mathrm{Y}-\mathrm{Q}$ carve and secondly, the point $\beta_{(\mathrm{t} \div \Delta \mathrm{t})}$ on the prolongation of the $\mathrm{Z}-\mathrm{V}$ curve; it will be recalled that $\Delta t$ should be chosen reasonably small for the approximations used to be valid.

Knowing $\mathrm{A}$, we can find $\mathrm{H}_{4 \mathrm{l}}$ and $\Delta \mathrm{V}$, as shown in fig. 8. Having found $\Delta Q$ from the accelerating head, point $\mathrm{B}_{\left(\mathrm{t}^{+} \Delta \mathrm{t}\right)}$ can be located on the $\mathrm{Z}-\mathrm{Q}$ curve, and thus the ordinate of $\beta(\mathrm{t} \Delta \mathrm{t})$ is linown; the abscissa of the same point is found from the value of $\Delta \mathrm{V}$, and the problem is thus solved. In this way the $\mathrm{Z}-\mathrm{V}$ curve can be plotted in successive steps (see fig. 9).

\section{(b) Second approximation :}

See fig. 10; the principle is of course the same as before.

\section{(c) Comments :}

1st. Comment. - The surge tank designed by the foregoing procedure is of continuously varying section. This should in practice be replaced by a structure comprising a certain number of expansion chambers of constant cross-section (see fig. 11).

2nd. Comment. - In designing throtlling type surge tanks, it is desirable lo define at the outset the $\mathrm{Z}-\mathrm{Q}$ curve pertaining to the pressure below the throttling inlet. The $\mathrm{Z}-\mathrm{Q}$ curve giving the free surface level in the structure may be immediately found by addition (or subtraction, as the case may be) of the head-loss in the throttled section, which is supposed known, and the problem thus reduces to that considered previously.

3rd. Comment. - Novable Axis : Non-instantaneous changes of turbine discharge can be treated with as much facility as instantaneous changes of regime.

4th. Comment. - Combination of Retrograde and Direct Methods of Design : Nothing prevents us from working backwards in the direct method of design; the graphical construction is carried out in the same way, be it for t increasing or decreasing.

5th. Comment. -- Other Combinations : Similarly, nothing prevents us from combining the normal method of design (in which the dimensions of the surge tank are supposed known in a certain range of altitude) with the direct method, in which the variation of base pressure, for example, is given above or below this range; it is required to complete the design of the structure by calculating the unknown dimensions. Other combinations are equally possible, and, as will be shown in illustrative examples below, also prove very useful in practice.

\section{III. - PRACTIGAL APPLICATIONS \\ OF AUTOMATIC METHODS OF SURGE TANK DESIGN}

The author's furnish several examples of structures which they have actually designed by automatic methods; it shonld be remarked that the cases given by no means exhaust the possibilities of automatic design, despite the fact that the author's restrict themselves here to the study of surge tanks of two types-throttling and multiple chamber structureswhich have some particalar advantages of their own.

A) Examples of the use of THE RETrograde Method

(a) 1st. Problem :

For an ordinary type of power scheme (single intake, single headrace tunnel and single surge tank), what are the sudden load increases from $\times$ to $100 \%$ which maly be permitted for different static levels at the intake?

The problem may be directly solved without any trial computations by the retrograde method (see fig. 15). Each given static level corresponds to a certain height difference $\left(\mathrm{H}, \mathrm{H}^{\prime}, \mathrm{H}^{\prime \prime}\right)$ between the base of the surge tank and the static level in question. All that has to be done is to start the graphical construction from the minimum permissible level (points $\mathrm{M}, \mathrm{M}^{\prime}, \mathrm{M}^{\prime \prime}$ ) and work backwards until the tunnel head-loss parabola is reached; the point of intersection gives the discharge corresponding to the permanent flow $(\mathrm{x} \%)$ existing in the head-race thnnel before the sudden taking-up of load.

A practical case is solved in "Epure $N^{\circ} 2$ " of the French text; in this instance, the surge tank consists in a cylindrical well of $17 \mathrm{~m}$. diameter, the base R.L. being $4.40 \mathrm{~m}$. The construction is carried out fully for the case of a siatic supply level at R.L. 47t.

\section{(b) 2nd. Problem :}

It is desired for practical reasons to construct a surge tank of two given shaft diameters, as illustrated to the right of fig. 16 . At what height should the transition from the one diameter to the other be located, in order to limit the maximum rise in level after a total shut-down (instantaneous or not) to a given value?

As shown in fig. 16, all that has to be done is to work from both limits of the Z-Q curve; the intersection of the two branches gives the required transition point.

(c) 3rd. Problem :

For how long does the free surface level in the surge tank remain below a certain given height during the surge causing the greatest drawn-down in the surge structure?

The graphical construction is commenced in both directions from $\mathrm{M}$, the lowest point reached during the draw-down (see fig. 1\%). The two branches of the graph are continued up as fal the given level and the $\Delta \mathrm{t}$ added up in the usual way. 
B) ESAMPLES OF THE USE OF THE DTRECT METHOD OF AUTOMATIC DESTGN

(a) 1st. Problem :

Design of the optimum form of throlling surge tank for a total instantaneous shut-down :- -

We suppose known

(i) the dimensions and head-loss of the head-race tunnel $\left(\mathrm{L}, \mathrm{f}, \mathrm{R}_{1}\right)$.

(ii) the maximum pressure at the base of the surge chamber, $Z_{m}$; this is supposed constant during the first rise in level in the structure aiter the shut-down. We thus have that the headloss in the throtling inlet, $\mathrm{R}_{2}$, is equal to $Z_{m}+R_{1}$ for the maximum discharge in the head-race tunnel.

Knowing $\mathrm{R}_{2}$, we can at once plot the parabola giving the water level in the surge tank as a function of the discharge flowing in the head-race tunnel; the direct method of automatic design then enables us to plot the $Z-\mathrm{V}$ curve without difficully and the problem is solved (see fig. 18).

Note. - It should be poinled out that throlling surge tanks of constant cross-section may be designed without any computation work by means of CALAME and GADEN's well-known solution curves (5); however, the above method of analysis may well prove useful in the case of very large structures, where the choice of a constant diameter tvonld entail an undue increase in to:ul volume (the point is, of course, that a constant diameter structure cannot ensure a rigorously constant base pressure, except, as we have seen, in the hypothetical case of zero head-loss in the head-race tunnel).

\section{Numerical example (See Epure No 3).}

The case studied is that of the sudden shul-down of a power station for which the maximum discharge is $100 \mathrm{~m} \% \mathrm{sec}$; the head-loss in the head-race tunnel for this flow is $1 / \mathrm{m}$., and that in the throttling intake to the stand pipe, $31 \mathrm{~m}$, for the same discharge.

The total volume of the structure, as found graphically, turns out to be $11,400 \mathrm{~m}: \mathrm{and}$ corresponds exacily to that given by the theoretical formula derived at the beginning of the paragraph on antomatic design by the "direct" method. CaLdum and GaDEx (5), on the other hand, give 12,900 m.-. a difference of $7.5 \%$, which may be worthwhile saving in some cases.

(b) 2nd. Problem :

Design of a multiple section surge tank by a combination of automatic (" divect") and ordinary ("indirect") methods.

Once again the dimensions and head-loss of the head-race tunnel are supposed known. In addition, we may suppose that practical reasons dictate the choice of an inside diameter for the central shafl, of the heights of the apper and lower expansion chambers and, finally, of the permissible limits of oscillation, of the free surface in the surge tank, $Z_{\mathrm{m}}$ and $Z_{\mathrm{m}}^{\prime}$ (see fig. 19).
Design for a shut-down

Seeing that the highest level attained in the surge lank under steady running condilions in the power station will certainly lie somewhere within the central shat of known diameter, there is no difficulty in starting off the graphical solution in the ordinary way and continaing up to the level $Z_{\mathrm{m}}-\mathrm{h}$, where his the desired height of the upper expansion chamber. On the $\mathrm{Z}-\mathrm{Q}$ diagram we can then trace a curve MN langent to the horizontal line passing through $\mathrm{N}$, the prescribed maximum level. The "direct" design procedure enables the rest of the $\mathrm{Z} \ldots \mathrm{Y} \mathrm{Y}$ curve to be plotled without difficulty (see fig. 19).

It should be remarked that there is an infinile number of curves passing through $\mathrm{M}$ and having a horizontal tangent at $\mathrm{N}$; most of these would however result in peculiarly shaped struclures. In practice, a parabola with apex at $\mathrm{N}$ is found to give a curve on the $\mathrm{Z}-\mathrm{V}$ graph which is practically straight; it is an easy matler to replace this curve by the straight line which it so closely resembles, the slope of which then gives the area in plan of the expansion chamber.

A special case of some interest occurs when the accelerating head $\mathrm{H}_{i}$ remains constant during the rise of level in the expansion chamber. This is so when the head-race tunnel head loss paraboia is identically similar to the carve $M N$, and, as will be shown, the $Z-\mathrm{V}$ curve over the range considered then tarns out to be precisely a straight line :-

The equations of motion are as follows :

$$
\begin{gathered}
-\frac{\mathrm{L}}{\mathrm{gf}} \frac{\mathrm{dQ}}{\mathrm{dt}}=Z+\mathrm{K}_{1}(\mathrm{Q})^{2} \\
Z=Z_{\mathrm{m}}-\mathrm{AQ}^{2} \\
\mathrm{Q}=\mathrm{F} \frac{\mathrm{d} Z}{\mathrm{dt}}
\end{gathered}
$$

where as before $\mathrm{Q}$ is the discharge in the head-race tannel and $\mathrm{A}$ is the parameter which defines the form of the parabola MN.

From (3) it follows that

$$
\begin{aligned}
F & =\frac{Q}{\frac{d Z}{d t}} \\
& =-\frac{1}{2 A \frac{\mathrm{dQ}}{\mathrm{dt}}} \quad \text { by }
\end{aligned}
$$

The value of the derivative may then be substilatad from (1), and we thus find that

$$
\begin{aligned}
\frac{1}{\mathrm{~F}} & =2 \frac{\mathrm{gf}}{\mathrm{I}} \mathrm{A}\left(\mathrm{Z}+\mathrm{K}_{1} \mathrm{Q}^{2}\right) \\
& \left.=2 \frac{\mathrm{g}^{\mathrm{f}}}{\mathrm{I}}\left[\mathrm{Z}_{\mathrm{m}} \mathrm{K}_{1}-\mathrm{Z}_{\mathrm{L}}-\mathrm{K}\right)\right] \quad \text { by }(2)
\end{aligned}
$$


Should $\mathrm{K}_{1}=\mathrm{A}$, the area of the expansion chamber is clearly constant.

\section{Design for a take-up of load.}

This is quite andogous to the preceding case (see (io. 19).

(c) 3rd. Problem :

The third problem trealed by the authors is one which they consider worthy of special attention.

Between the two extremes types of surge tankthrolling inlet and multiple chamber structuresthere is a whole range of intermediale lypes having both throttling inlets and expansion chambers; such hybrid structures have certain real advantages over the lwo extreme lypes, as they combine the good points of both. To make this clear, we shall consider a practical example :-

Suppose that a surge lank is to be designed for a power scheme for which $\mathrm{L}$, $\mathrm{f}$ and $\mathrm{Q}$ are already fixed, and for which the level of the headrace tumnel is already decided on beforehand (as is unfortunalely often the case); the elevation of the bollom of the surge tank is thus given. Sintilarly, the maximum allowable pressure rise in the headrace tunnel at the base of the surge structurc is also specified.

For such a project it often happens that neither. of the limiting types of structure already mentioned can be adopted, for one of the following reasons: :-...

Structure with optimum head-loss in the throttlins inlet.

(i) Because the central shat atameter is too greal; this is a drawback for construction.

(ii) Because R. is too large and would cause damage to the head-nace Innnel in the event of a sudden shut-down of the power plant (T).

(iii) Becanse slow, or partial, closure of the lawbines results in a greater rise in waler level than " complete, instantaneous shut-down.

Multiple chamber structure

(i) Because the least change in the flow passing through the lubines causes the free surface in the surge lank to oscillate between the upper and lower expansion chambers; this is detrimental to good turbine regulation and, above all, is angerous for the head-race tunnel. It is true that this can be partly remedied by increasing the diameter of the central shaft, but this can be done only at the expense of a considerable increase in total volume.

In these circumstances the choice of an intermediate type of structure is indicaled. One assumes at the outset :-

(a) a value of the head loss R., al the throlling inlet compatible with the strenglh of the tamnel lining.

(b) the cross-sectional area of the central shaft; this may be determined by the Troms condition (giving the minimum size of shat for stable tarbine requlation), by conslraction requirements or by some olher consideration.
The "direct" (automalic) and "indirect" (normal analytical) methods of design may then be used in conjunction to solve the problem, one graphical construction being required for the most severe shutdown and another for the putting into operation of the plant under the most severe service conditions.

(1) Computation of a total shut-down (gradual or instantaneous) :

As in the case of the pure throttling type of structure, the most advantageous design is that ensuring a constant base pressure $Z_{\mathrm{m}}$ below the throtlling inlet as soon as the free surface reaches the invert of the upper expansion chamber. It is under'stood of course that the actual value of $\mathrm{Z}_{\mathrm{n}}$ is given as part of the design data.

As has been shown above, the most logical procedure is to construct the $Z-Q$ carve pertaining to the part of the rise which takes place in the upper expansion chamber in the form of a parabola found by subtracting $\mathrm{R}_{2}=\mathrm{K}_{2} \mathrm{Q}^{2}$ from the given level $Z_{\mathrm{m}}$. The analysis already given is then applicable and the area of the expansion chamber can be immediately calculated. It then remains to fix the precise height of the expansion chamber, and this can be done by the normal, "indirect" construction; starting from the point corresponding to the full turbine discharge under constant ranning conditions the operational curve is plotted upwards to cut the parabola already spoken of (see fig. 20 ).

(2) Computation for a sudden throw-on of load :

It is presumed that the most severe case to be considered under this heading is specified beforehand (e.g., a gradual or instarlaneous increase in tarbine discharge from $x \% 10100 \%)$, and that the value of the head-loss in the throttling inlet to the surge lank is also knomm, shomld this happen to be dissymmetrical.

As for the formen compulation, the construction is carried out by combining the " divect" and "indirect" methods (see fig. 21).

Comments :

Firstly, let it be recalled that, even should the $\mathrm{Z}-\mathrm{V}$ carves for the expansion chambers depart somewhat from the straight line form, it is generaliy adequale to replace them by a broken line as shown in fig. 22. The donble expansion chambers shown in the illustration are in actual fact very convenient from the constructional standpoint.

Secondly, it should be borne in mind that a " hybrid" lype of surge struclare may, or may not, be of larger volume than a constant section throtling type; in the former case, the difference is well compensated by a more gradual pressure build-up in the head-race tunnel following on a sudden shut-down of the tarbines, as well as by a greater ease of construction.

Practical Example (see Epure $\left.\mathrm{N}^{\circ} 4\right)$

The installation is the same as that sludied in "Epture $N^{\circ} 3 "$-the lenglh of the head-racie tunnel 
is 16,144 metres, its cross-sectional area 31.1 square metres and the maximum discharge passing through the turbines is $100 \mathrm{~m}^{3} / \mathrm{sec}$. For the present problem it is supposed that the central shaft measures $17 \mathrm{~m}$. in diameter, and that the value of $\mathrm{R}_{2}$ for the maximum discharge just specified is $15 \mathrm{~m}$. (instead. of 31). On the other hand, the maximum base pressure below the throttling inlet is still equal to $17 \mathrm{~m}$., as in the former case, since the maximum lise of level in the structure remains the same.

The graphical construction gives $12,480 \mathrm{~m}^{3}$ as the total required volume of the structure; replacement of the variable section expansion chambers found theoretically from the diagram by double section chambers would in this particular case increase the tolal volume by about $1 \%$. The volume required for the plain throttling type with a constant section shaft was previously found to be $12,200 \mathrm{~m}^{3}$; counting the additional $1 \%$ just mentioned, the difference between the two designs works out at about $3 \%$.

Thus, it turns out in this particular case that the head-loss in the throtting inlet can be reduced from 31 to $15 \mathrm{~m}$. without any appreciable increase in total volume; it would even be possible to redace the margin of 3 \% found above to zero by the choice of a smaller diameter for the central shaft.

\section{REFERERENCES}

1. .... Funglish readers should refer to, pp. $868-873$ of «Hydraulies Structures ». (A.S.M.E., 1937 ).

2. - T. Schrmanx : Zeichnerisches Vertahren zu Darstellung der Schwingungen in Kammer- und Ueberfallwasserschlössern ». Wasserlifaft and Wasserwirtschaft, March 16th, 1942.

3. - E. Der Ferres: «Acorgimenti pratici per la determinazione grafica delle oscillazioni nelle camera di equilibrio di qualsiasi lipo o. Tínergia Elettrica, nos $4-5,1947$.

4. - V. Goumkis : « Méthode genérale de calcul des chambres d'écuilibre alimentées par 2 canaux d'amenéc $\gg$. La Houlle Blanche, n" 3, ma-june, 1948.

5. J. Calame and D. Garen: "Théorie des chambles d'équilibre ». Gaththiers-Villars, Paris, 1926.

6. - L. Escande: \& Méhodes nouvelles pour le calcul des chambres d'équilibre \%. Danod, Paris, 1950.

7. -... Ponsar : "Sur les coups de belier donde pouvant che crés par le diaphragme placé à la base d'une cheminée d'équilibre $\approx$. La Honille Blanche, n" $2,1950$.

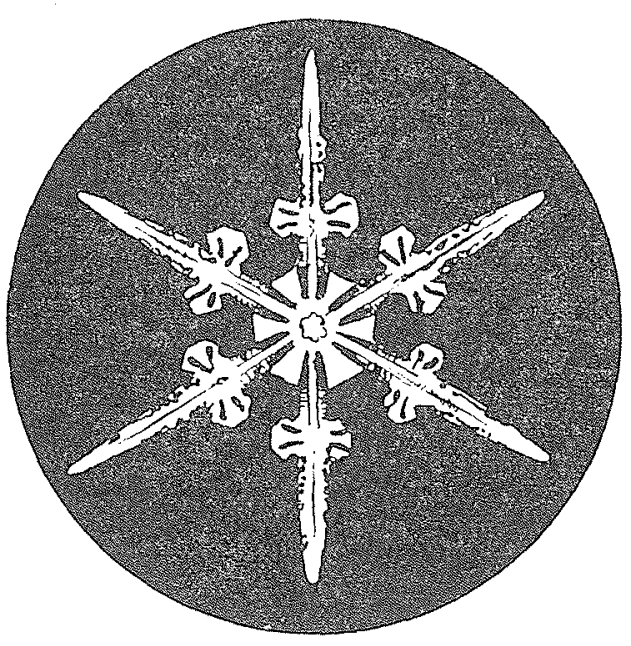

